ESAIM: PROCEEDINGS AND SURVEYS, February 2015, Vol. 49, p. 130-157

Samira EL YACOUBI, Larbi AFIFI, El Hassan ZERRIK, Abdessamad TRIDANE, Editors

\title{
REGIONAL CONTROLLABILITY OF DISTRIBUTED BILINEAR SYSTEMS *
}

\author{
E.H. ZERRIK ${ }^{1}$ AND R. LARHRISSI ${ }^{2}$
}

\begin{abstract}
In this paper, we consider the problem of optimal regional controllability of a distributed bilinear system evolving on $\Omega$. The question is to obtain a control with minimum energy that drives such a system from an initial state to a final state close to a desired one in finite time, only on a subregion $\omega$ of $\Omega$. Our purpose is to prove that a regional optimal control exists and characterized in both bounded and unbounded cases. The obtained results are successfully illustrated by simulations.

Résumé. Dans cet article nous considérons le problème de la contrôlabilité régionale optimale d'un système distribué bilinéaire évoluant sur un domaine spatial $\Omega$. La question est d'obtenir un contrôle à énergie minimale qui conduit un tel système d'un état initial vers un état final proche d'un état désiré uniquement sur sur une région $\omega$ de $\Omega$. On montre qu'un tel contrôle existe et ceractérisé dans les cas borné et non borné. Les résultats obtenus sont illustrés avec succès par des simulations.
\end{abstract}

\section{INTRODUCTION}

Bilinear systems involve products of state and control, which means that they are linear in state and linear in control but not jointly linear in state and control. The interest of these systems lies in the fact that many natural and industrial processes have intrinsically bilinear structure. This is the cases of furnaces for heating metal slabs or heat exchangers, aircrafts and robot arms or energy transmission lines. Clearly such models involve using sophisticated mathematical methods, which requires to describe the process more accurately and to implement more effective control strategies.

Let $\Omega \subset \mathbb{R}^{n}(n=1,2,3)$ be a spatial domain with regular boundary $\partial \Omega$. For $T>0$ and $\left.\Pi=\Omega \times\right] 0, T[$, $\Sigma=\partial \Omega \times] 0, T[$, this class of systems may be described by the equation

$$
\begin{cases}\frac{\partial z(x, t)}{\partial t}=A z(x, t)+u(t) B z(x, t) & \Pi \\ z(x, 0)=z^{0}(x) & \Omega\end{cases}
$$

where $A$ is the generator of a strongly continuous semigroup $(S(t))_{t \geq 0}$ on the state space $Z=: L^{2}(\Omega)$ endowed with its natural inner product $<,>$ and the corresponding norm $\|\|, B:. Z \rightarrow Z$ is a linear bounded operator, and $u \in L^{2}[0, T]$ is a control. The main result on controllability of system (1) is due to the pioneering work, which shows that under the above-mentioned conditions, the mild solution $z_{u}$ of (1) associated to the control $u$ exists and the set of reachable states from an initial state $z_{0}$ is of dense complement in the state space. This makes exact controllability difficult to be achieved and the most obtained results are established for particular

\footnotetext{
* MACS Team, Faculty of Sciences, University Moulay Ismail. Meknes- Morocco.

1 zerrik3@yahoo.fr

2 r.larhrissi@gmail.com
} 
bilinear systems (Ball et al., 1982; Joshi, 2005; Lenhart and Liang, 2000; Khapalov, 2002 see [3, 6, 10,15, 22, 24]).

The concept of regional controllability for distributed systems, developed by El jai and Zerrik, reformulated the classical notion of controllability and made possible controllability of such systems only on a subregion of the system's spatial evolution domain. This concept finds its application in many real world problems. For example the physical problem which concerns tunnel furnace where one has to maintain a prescribed temperature only in a subregion of the furnace. Also there exist systems which are controllable on some subregion $\omega \subset \Omega$ but not controllable in the whole domain $\Omega$ and that controlling regionally a system is cheaper than controlling it in the whole domain (see [18]). The reader may find an interesting development for this topic in works developed by several researchers of our net work since 1993, for linear and semi linear systems, particularly characterizations of control that achieves regional controllability with minimum energy (see [21]).

In this paper we discuss an extension of previous works on regional controllability for linear and semi linear systems to bilinear one. More precisely for system (1) defined on a spatial domain $\Omega$, a non empty subset $\omega \subset \Omega$, with positive Lebesgue measure and a desired state $z_{d}$ in $L^{2}(\omega)$, the problem of regional controllability for (1) consists in finding a control function with minimum energy in an appropriate controls space that steers the system (1) from $z_{0}$ to a final state close to $z_{d}$ on $\omega$ at time $T$.

This problem may be stated as follows:

$$
\left\{\begin{array}{l}
\text { Finding } u \in V \subseteq L^{2}[0, T] \text { which minimizes }\|u\|_{L^{2}[0, T]}^{2} \\
u \in U_{a d}(\omega)
\end{array}\right.
$$

while

$$
U_{a d}(\omega)=\left\{u \in V \subseteq L^{2}[0, T]:\left\|\chi_{\omega} z_{u}(T)-z_{d}\right\|_{L^{2}(\omega)} \text { is minimum }\right\}
$$

We discuss both cases :

$$
V=L^{2}[0, T] \quad \text { and } \quad V=U_{M}=\left\{u \in L^{\infty}([0, T]) \mid-M \leq u(t) \leq M\right\}
$$

To characterize an optimal control solution of the problem (2), we propose an approach based on quadratic cost control problem, which involves the minimization of the control norm and the final state error, this is the aim of this paper which is organized as follow :

In the second section, we consider the problem (2) with unbounded controls and we give a characterization of an optimal one. In the third section, the problem (2) is examined with bounded controls and we show the existence of an optimal control solution of the problem (2) by solving an optimality system. The obtained results are successfully illustrated by simulations in the both cases.

\section{UnBOUNDED CONTROLS CASE}

In this section, we consider the problem (2) with $V=L^{2}[0, T]$. A solution of quadratic cost control problem associated to (2) allows us to give a characterization of an optimal control solution of the problem (2). Then we show that under supplementary conditions, the uniqueness may be ensured. Also we give illustrations by simulations. 


\subsection{Regional quadratic control problem}

Fix $T>0$ and let associate to (2) the problem :

$$
\min _{u \in L^{2}[0, T]} J_{\epsilon}(u)
$$

with

$$
J_{\epsilon}(u)=\left\|\chi_{\omega} z_{u}(T)-z_{d}\right\|_{L^{2}(\omega)}^{2}+\epsilon \int_{0}^{T} u^{2}(t) d t, \epsilon>0 .
$$

Let us first show the following results

Proposition 1.1. (1) For $u \in L^{2}[0, T]$ and $h \in L^{2}[0, T], \forall t \in[0, T]$, we have $\left\|z_{u+h}(t)-z_{u}(t)\right\| \leq C\|h\|$

(2) There exists $u^{*} \in L^{2}[0, T]$ such that

$$
J_{\varepsilon}\left(u^{*}\right)=J^{*}=\min _{v \in L^{2}[0, T]} J_{\varepsilon}(v)
$$

(3) Let $y(t)=\int_{0}^{t} U(t, s) h(s) B z_{u}(s) d s$ then $\left\|z_{u+h}(t)-z_{u}(t)-y(t)\right\|=o(\|h\|)$, as $h \rightarrow 0$ where $(U(t, s))_{t \geq s}$ is the evolution operator generated by $A+u B$.

Proof. (1) We have

$$
z_{u+h}(t)-z_{u}(t)=\int_{0}^{t} S(t-s) u(s) B\left(z_{u+h}(s)-z_{u}(s)\right) d s+\int_{0}^{t} S(t-s) h(s) B z_{u+h}(s) d s .
$$

Using the boundedness of the semigroup $(S(t))_{t \geq 0}$ in all finite interval of $[0, T]$, i.e., $\exists M>0$ such that $\|S(t)\| \leq M, \forall t \in[0, T]$, we have

$$
\left\|z_{u+h}(t)-z_{u}(t)\right\| \leq M\|B\| \times\left(\int_{0}^{t}\left[|u(s)|\left\|z_{u+h}(s)-z_{u}(s)\right\|+|h(s)|\left\|z_{u+h}(s)\right\|\right] d s\right)
$$

and

$$
\left\|z_{u+h}(t)\right\| \leq M \times\left(\left\|z_{0}\right\|+\|B\| \int_{0}^{t}|u(s)+h(s)|\left\|z_{u+h}(s)\right\| d s\right) .
$$

Using the Gronwall inequality twice, we obtain $\left\|z_{u+h}(t)\right\| \leq k_{1}$, and

$$
\left\|z_{u+h}(t)-z_{u}(t)\right\| \leq M\|B\| \times\left(\int_{0}^{t}|u(s)|\left\|z_{u+h}(s)-z_{u}(s)\right\| d s+k_{1} \int_{0}^{t}|h(s)| d s\right)
$$

and again by Gronwall inequality, we obtain $\left\|z_{u+h}(t)-z_{u}(t)\right\| \leq C\|h\|$.

(2) The set $E=\left\{J_{\varepsilon}(u) \mid u \in L^{2}[0, T]\right\}$ is nonempty and bounded from below, so the lower bound $J^{*}$ exists. Let the sequel $\left(u_{n}\right)$ in $L^{2}[0, T]$ be such that $\lim _{n \rightarrow+\infty} J_{\varepsilon}\left(u_{n}\right)=J^{*}$, we have

$$
\varepsilon \int_{0}^{T} u_{n}^{2}(t) d t \leq J_{\varepsilon}\left(u_{n}\right)
$$

so, $\left(u_{n}\right)_{n \geq 0}$ is bounded, therefore we can extract a subsequence denoted $\left(u_{n_{k}}\right)$ which converges weakly to $u^{*}$ in $L^{2}[0, T]$. This implies that $z_{u_{n_{k}}}$ converges to $z_{u^{*}}$ strongly in $\mathcal{C}(0, T ; Z)$ (see [3]), hence

$$
J_{\varepsilon}\left(u^{*}\right) \leq \lim _{n \rightarrow+\infty} \inf J_{\varepsilon}\left(u_{n}\right)=J^{*} \leq J_{\varepsilon}\left(u^{*}\right) .
$$


(3) Since $u \in L^{2}[0, T]$ and $B$ is a linear bounded operator on $Z$, the operator $A+u B \in L^{1}[0, T ; D(A)]$, then $A+u(t) B$ generates an evolution operator $(U(t, s))_{t \geq s}([19])$.

So $y(t)=\int_{0}^{t} U(t, s) h(s) B z_{u}(s) d s$ is well defined.

Let $Y(t)=z_{u+h}(t)-z_{u}(t)-y(t)$, we can write

$$
\begin{aligned}
Y(t) & =\int_{0}^{t} S(t-s) u(s) B Y(s) d s \\
& +\int_{0}^{t} S(t-s) h(s) B\left(z_{u+h}(s)-z_{u}(s)\right) d s \\
& +\int_{0}^{t} S(t-s) h(s) B z_{u}(s) d s \\
& +\int_{0}^{t} S(t-s) u(s) B y(s) d s-y(t) .
\end{aligned}
$$

Let

$$
K(t)=\int_{0}^{t} S(t-s) h(s) B z_{u}(s) d s+\int_{0}^{t} S(t-s) u(s) B y(s) d s-y(t)
$$

then, for $z_{0} \in D(A)$,

$$
\begin{aligned}
\dot{K}(t) & =A \int_{0}^{t} S(t-s) h(s) B z_{u}(s) d s+h(t) B z_{u}(t)+A \int_{0}^{t} S(t-s) u(s) B y(s) d s \\
& +u(t) B y(t)-\dot{y}(t) .
\end{aligned}
$$

Since $\dot{y}(t)=(A+u(t) B) y(t)+h(t) B z_{u}(t)$ and $y(0)=0$, then

$$
y(t)=\int_{0}^{t} S(t-s) h(s) B z_{u}(s) d s+\int_{0}^{t} S(t-s) u(s) B y(s) d s
$$

which shows that $\dot{K}(t)=0$, and since $K(0)=0$, it follows that $K(t)=0, \forall t \in[0, T]$.

Then we have

$$
Y(t)=\int_{0}^{t} S(t-s) u(s) B Y(s) d s+\int_{0}^{t} S(t-s) h(s) B\left(z_{u+h}(s)-z_{u}(s)\right) d s
$$

and

$$
\|Y(t)\| \leq M\|B\|\left(\int_{0}^{t}|u(s)|\|Y(s)\| d s+\int_{0}^{t}|h(s)|\left\|z_{u+h}(s)-z_{u}(s)\right\| d s\right)
$$

by property 1 , we have

$M\|B\| \int_{0}^{t}|h(t)|\left\|z_{u+h}(s)-z_{u}(s)\right\| d s \leq k_{1}\|h\|^{2}, k_{1} \in \mathbb{R}$.

By Gronwall inequality, we obtain

$$
\|Y(t)\| \leq k_{2}\|h\|^{2}, k_{2} \in \mathbb{R}, \text { that is }\|Y(t)\|=o(\|h\|),
$$

and by the density of $D(A)$ in $Z$, we have the above inequality in $Z$.

Now, the solution of problem (3) is characterized by the following result. 
Theorem 1.2. A control which minimizes the problem (3) is given by

$$
u(t)=-\frac{1}{\epsilon}\left\langle B z(t), P(t) z(t)-U^{*}(T, t) \chi_{\omega}^{*} z_{d}\right\rangle
$$

where $P$ is the self-adjoint and non negative operator, solution of the following equation

$$
\left\{\begin{array}{l}
\frac{d}{d t}\langle P(t) y, z\rangle+\langle P(t) y,(A+u(t) B) z\rangle \\
+\langle(A+u(t) B) y, P(t) z\rangle=0, \forall y, z \in D(A) \\
P(T)=\chi_{\omega}^{*} \chi_{\omega} .
\end{array}\right.
$$

$U^{*}(t, s)$ is the adjoint operator of $U(t, s)$ and $\chi_{\omega}^{*}$ is the adjoint operator of $\chi_{\omega}$.

The minimum is given by

$$
J_{\varepsilon}(u)=\left\langle P(0) z_{0}, z_{0}\right\rangle+2\left\langle\chi_{\omega} U(T, 0) z_{0}, z_{d}\right\rangle+\left\|z_{d}\right\|_{L^{2}(\omega)}^{2}+\varepsilon \int_{0}^{T} u^{2}(t) d t .
$$

Proof. Using property 3 of the previous proposition, we have

$$
z_{u+h}(t)=z_{u}(t)+y(t)+o(h)
$$

then we obtain

$$
\left\langle\chi_{\omega} z_{u+h}(t)-z_{d}, \chi_{\omega} z_{u+h}(t)-z_{d}\right\rangle=\left\langle\chi_{\omega} z_{u}(t)-z_{d}, \chi_{\omega} z_{u}(t)-z_{d}\right\rangle+2\left\langle\chi_{\omega} z_{u}(t)-z_{d}, \chi_{\omega} y(t)\right\rangle+o(\|h\|)
$$

then

$$
J_{\varepsilon}(u+h)-J_{\varepsilon}(u)=2\left\langle\chi_{\omega} z_{u}(T)-z_{d}, \chi_{\omega} y(T)\right\rangle+2 \varepsilon \int_{0}^{T} u(t) h(t) d t+o(\|h\|) .
$$

Let $\Lambda$ be the operator defined by :

$$
\begin{aligned}
\Lambda: \quad L^{2}[0, T] & \rightarrow \quad L^{2}\left[0, T, L^{2}(\omega)\right] \\
v(.) & \rightarrow \Lambda v(.)=\chi_{\omega} U(T, .) v(.) B z_{u}(.) .
\end{aligned}
$$

Since

then we obtain

$$
y(T)=\int_{0}^{T} U(T, s) h(s) B z_{u}(s) d s
$$

$$
J_{\varepsilon}(u+h)-J_{\varepsilon}(u)=2 \int_{0}^{T}\left[\left\langle\chi_{\omega} z_{u}(T)-z_{d}, \Lambda h(t)\right\rangle+\varepsilon u(t) h(t)\right] d t+o(\|h\|) .
$$

Thus, the differential of $J_{\varepsilon}$ at $u$ :

$$
d J_{\varepsilon}(u) h=2 \int_{0}^{T}\left[\left\langle\Lambda^{*}\left(\chi_{\omega} z_{u}(T)-z_{d}\right), h(t)\right\rangle+\varepsilon u(t) h(t)\right] d t .
$$

Then $d J_{\varepsilon}(u) h=0, \forall h \in L^{2}[0, T]$ is equivalent to

$$
u(t)=-\frac{1}{\varepsilon} \Lambda^{*}\left(\chi_{\omega} z_{u}(T)-z_{d}\right) .
$$


Also we have

$$
\Lambda^{*} v(t)=z_{u}^{*}(t) B^{*} U^{*}(T, t) \chi_{\omega}^{*} v(t)
$$

which gives

$$
u(t)=-\frac{1}{\varepsilon} z_{u}^{*}(t) B^{*} U^{*}(T, t)\left(\chi_{\omega}^{*} \chi_{\omega} z_{u}(T)-\chi_{\omega}^{*} z_{d}\right) .
$$

Let us consider the following non negative and self-adjoint operator

$$
P(t) z=U^{*}(T, t) \chi_{\omega}^{*} \chi_{\omega} U(T, t) z, \quad \forall z \in D(A) .
$$

Since $z_{u}(t)=U(t, 0) z_{0}$, we have $P(t) z_{u}(t)=U^{*}(T, t) \chi_{\omega}^{*} \chi_{\omega} z_{u}(T)$, then we obtain (5).

Let us show that $P(t)$ satisfies the equation (6).

We have

$$
\frac{\partial U}{\partial s}(t, s) z=-U(t, s)(A+u(s) B) z, \forall z \in D(A),
$$

then, $\forall y, z \in D(A)$ we obtain

$\frac{d}{d t}\left\langle U^{*}(T, t) \chi_{\omega}^{*} \chi_{\omega} U(T, t) y, z\right\rangle=-\left\langle\chi_{\omega} U(T, t)(A+u(t) B) y, \chi_{\omega} U(T, t) z\right\rangle-\left\langle\chi_{\omega} U(T, t) y, \chi_{\omega} U(T, t)(A+u(t) B) z\right\rangle$,

which shows the right part of (6).

Now, using

$$
z_{u}(t)=U(t, 0) z_{0},\left\langle\chi_{\omega} z_{u}(T), \chi_{\omega} z_{u}(T)\right\rangle=\left\langle P(0) z_{0}, z_{0}\right\rangle
$$

thus

$$
J_{\varepsilon}(u)=\left\langle P(0) z_{0}, z_{0}\right\rangle+2\left\langle\chi_{\omega} U(T, 0) z_{0}, z_{d}\right\rangle+\left\|z_{d}\right\|_{L^{2}(\omega)}^{2}+\varepsilon \int_{0}^{T} u^{2}(t) d t .
$$

Remark 1.3. The equation (6) has a unique solution (see [6]).

If $u_{\epsilon}$ denotes the solution of (3) and $z_{\epsilon}$ the associated state of (1), the following result will be useful for the sequel of the paper.

Proposition 1.4. (1) The sequence $\left(J_{\epsilon}\left(u_{\epsilon}\right)\right)_{\epsilon>0}$ is increasing with respect to $\epsilon$.

(2) The sequence $\left(\int_{0}^{T} u_{\epsilon}^{2}(t) d t\right)_{\epsilon>0}$ is decreasing with respect to $\epsilon$.

(3) The sequence $\left(\left\|\chi_{\omega} z_{\epsilon}(T)-z_{d}\right\|_{L^{2}(\omega)}^{2}\right)_{\epsilon>0}$ is increasing with respect to $\epsilon$. and $\forall \epsilon>0$

$$
\left\|\chi_{\omega} z_{\epsilon}(T)-z_{d}\right\|_{L^{2}(\omega)} \leq\left\|\chi_{\omega} S(T) z_{0}-z_{d}\right\|_{L^{2}(\omega)}
$$

In particular, there exists a subsequence of $\left(\chi_{\omega} z_{\epsilon}(T)-z_{d}\right)_{\epsilon>0}$ which converges weakly in $L^{2}(\omega)$. 
Proof. Let $0<\epsilon_{1}<\epsilon_{2}$, using consecutively the optimality of $u_{\epsilon_{1}}$ for $J_{\epsilon_{1}}$ and the optimality of $u_{\epsilon_{2}}$ for $J_{\epsilon_{2}}$, we have:

This implies that

$$
\begin{aligned}
J_{\epsilon_{1}}\left(u_{\epsilon_{1}}\right) & =\left\|\chi_{\omega} z_{\epsilon_{1}}(T)-z_{d}\right\|_{L^{2}(\omega)}^{2}+\epsilon_{1} \int_{0}^{T} u_{\epsilon_{1}}^{2}(t) d t \\
& \leq\left\|\chi_{\omega} z_{\epsilon_{2}}(T)-z_{d}\right\|_{L^{2}(\omega)}^{2}+\epsilon_{1} \int_{0}^{T} u_{\epsilon_{2}}^{2}(t) d t \\
& \leq\left\|\chi_{\omega} z_{\epsilon_{2}}(T)-z_{d}\right\|_{L^{2}(\omega)}^{2}+\epsilon_{2} \int_{0}^{T} u_{\epsilon_{2}}^{2}(t) d t \\
& \leq\left\|\chi_{\omega} z_{\epsilon_{1}}(T)-z_{d}\right\|_{L^{2}(\omega)}^{2}+\epsilon_{2} \int_{0}^{T} u_{\epsilon_{1}}^{2}(t) d t .
\end{aligned}
$$

From (7), we obtain

$$
J_{\epsilon_{1}}\left(u_{\epsilon_{1}}\right) \leq J_{\epsilon_{2}}\left(u_{\epsilon_{2}}\right) .
$$

and then

$$
J_{\epsilon_{2}}\left(u_{\epsilon_{2}}\right)-J_{\epsilon_{1}}\left(u_{\epsilon_{2}}\right) \leq J_{\epsilon_{2}}\left(u_{\epsilon_{1}}\right)-J_{\epsilon_{1}}\left(u_{\epsilon_{1}}\right)
$$

$$
\int_{0}^{T} u_{\epsilon_{2}}^{2}(t) d t \leq \int_{0}^{T} u_{\epsilon_{1}}^{2}(t) d t
$$

Hence, $\left\|\chi_{\omega} z_{\epsilon_{1}}(T)-z_{d}\right\|_{L^{2}(\omega)}^{2} \leq\left\|\chi_{\omega} z_{\epsilon_{2}}(T)-z_{d}\right\|_{L^{2}(\omega)}^{2}$ which shows statements 1., 2. and the first part of 3 . For $u=0$, we have $z_{u}(T)=S(T) z_{0}$ and, $\forall \epsilon>0$,

$$
\left\|\chi_{\omega} z_{\epsilon}(T)-z_{d}\right\|_{L^{2}(\omega)}^{2}+\epsilon \int_{0}^{T} u_{\epsilon}(t)^{2} d t \leq\left\|\chi_{\omega} S(T) z_{0}-z_{d}\right\|_{L^{2}(\omega)}^{2} .
$$

Then

$$
0 \leq\left\|\chi_{\omega} z_{\epsilon}(T)-z_{d}\right\|_{L^{2}(\omega)}^{2} \leq\left\|\chi_{\omega} S(t) z_{0}-z_{d}\right\|_{L^{2}(\omega)}^{2}, \forall \epsilon>0 .
$$

Finally, $\left(\left\|\chi_{\omega} z_{\epsilon}(T)-z_{d}\right\|_{L^{2}(\omega)}\right)_{\epsilon>0}$ is bounded. Then we can extract a subsequence of $\left(\chi_{\omega} z_{\epsilon}(T)-z_{d}\right)_{\epsilon>0}$ which converges weakly in $L^{2}(\omega)$.

\subsection{Regional minimum energy control problem}

Here let us go back to problem (2), and consider the set

$$
R(T)=\bigcup_{u \in L^{2}[0, T]}\left\{z_{u}(T)\right\}
$$

of reachable states at time $T$ from $z_{0}$. We have the main result.

Theorem 1.5. Let $u_{\epsilon}$ be a solution of (3) and assume that $U_{a d}(\omega)$ is nonempty, then we have

$$
u_{\epsilon} \rightarrow u^{\star} \text { as } \epsilon \rightarrow 0 \text { in } L^{2}[0, T]
$$

and

$$
\chi_{\omega} z_{\epsilon} \rightarrow \chi_{\omega} z_{u^{\star}} \text { in } C\left([0, T] ; L^{2}(\omega)\right) .
$$

Moreover $u^{\star}$ is a solution of (2).

Proof. Using the optimality of $u_{\epsilon}$ for $J_{\epsilon}$ we have, $\forall \epsilon>0, u \in L^{2}[0, T] J_{\epsilon}\left(u_{\epsilon}\right) \leq J_{\epsilon}(u)$, i.e.,

$$
\left\|\chi_{\omega} z_{\epsilon}(T)-z_{d}\right\|_{L^{2}(\omega)}^{2}+\epsilon \int_{0}^{T} u_{\epsilon}^{2}(t) d t \leq\left\|\chi_{\omega} z_{u}(T)-z_{d}\right\|_{L^{2}(\omega)}^{2}+\epsilon \int_{0}^{T} u^{2}(t) d t .
$$


$U_{a d}(\omega)$ is nonempty means that there exists $v \in L^{2}[0, T]$, such that

$$
\left\|\chi_{\omega} z_{v}(T)-z_{d}\right\|_{L^{2}(\omega)}^{2}=\min _{z \in R(T)}\left\|\chi_{\omega} z-z_{d}\right\|_{L^{2}(\omega)}^{2} .
$$

So, we have

$$
\forall u \in U_{a d}(\omega) \quad \int_{0}^{T} u_{\epsilon}^{2}(t) d t \leq \int_{0}^{T} u^{2}(t) d t, \quad \forall \epsilon>0 .
$$

Therefore, we can extract a subsequence also denoted $\left(u_{\epsilon}\right)_{\epsilon>0}$ such that $u_{\epsilon} \rightarrow u^{\star}$ weakly in $L^{2}[0, T]$ and $z_{\epsilon} \rightarrow z_{u^{\star}}$ strongly in $C([0, T] ; Z)$ as $\epsilon \rightarrow 0$ (see [3]), and this implies, $\chi_{\omega} z_{\epsilon} \rightarrow \chi_{\omega} z_{u^{\star}}$ strongly in $C\left([0, T] ; L^{2}(\omega)\right)$.

And, since $u_{\epsilon} \rightarrow u^{\star}$ weakly in $L^{2}[0, T]$, by the lower semi continuity of the norm, we have

$$
\lim _{\epsilon \rightarrow 0} \inf \int_{0}^{T} u_{\epsilon}^{2}(t) d t \geq \int_{0}^{T} u^{\star 2}(t) d t \quad \text { and } \quad \lim _{\epsilon \rightarrow 0} \inf J_{\epsilon}\left(u_{\epsilon}\right) \geq\left\|\chi_{\omega} z_{u^{\star}}(T)-z_{d}\right\|_{L^{2}(\omega)}^{2} .
$$

Moreover, $J_{\epsilon}\left(u_{\epsilon}\right) \leq J_{\epsilon}(u) \forall u \in L^{2}[0, T]$ so,

$$
\lim _{\epsilon \rightarrow 0} \sup J_{\epsilon}\left(u_{\epsilon}\right) \leq\left\|\chi_{\omega} z_{u}(T)-z_{d}\right\|_{L^{2}(\omega)}^{2} \forall u \in L^{2}[0, T]
$$

and in particular,

$$
\begin{aligned}
\lim _{\epsilon \rightarrow 0} \sup J_{\epsilon}\left(u_{\epsilon}\right) & \leq\left\|\chi_{\omega} z_{v}(T)-z_{d}\right\|_{L^{2}(\omega)}^{2} \\
& \leq\left\|\chi_{\omega} z_{u^{\star}}(T)-z_{d}\right\|_{L^{2}(\omega)}^{2} \\
& \leq \lim _{\epsilon \rightarrow 0} \inf J_{\epsilon}\left(u_{\epsilon}\right)
\end{aligned}
$$

hence,

$$
\begin{aligned}
\lim _{\epsilon \rightarrow 0} J_{\epsilon}\left(u_{\epsilon}\right) & =\lim _{\epsilon \rightarrow 0}\left\|\chi_{\omega} z_{\epsilon}(T)-z_{d}\right\|_{L^{2}(\omega)}^{2} \\
& =\left\|\chi_{\omega} z_{u^{\star}}(T)-z_{d}\right\|_{L^{2}(\omega)}^{2} \\
& =\left\|\chi_{\omega} z_{v}(T)-z_{d}\right\|_{L^{2}(\omega)}^{2} .
\end{aligned}
$$

Thus

$\lim _{\epsilon \rightarrow 0}\left\|\chi_{\omega} z_{\epsilon}(T)-z_{d}\right\|_{L^{2}(\omega)}^{2}=\min _{z \in R(T)}\left\|\chi_{\omega} z-z_{d}\right\|_{L^{2}(\omega)}^{2}$, and $u^{\star} \in U_{a d}(\omega)$.

Furthermore,

(11) gives

$$
\left\|\chi_{\omega} z_{\epsilon}(T)-z_{d}\right\|_{L^{2}(\omega)}^{2}+\epsilon \int_{0}^{T} u_{\epsilon}^{2}(t) d t \leq\left\|\chi_{\omega} z_{u^{\star}}(T)-z_{d}\right\|_{L^{2}(\omega)}^{2}+\epsilon \int_{0}^{T} u^{\star 2}(t) d t
$$

$$
\int_{0}^{T} u_{\epsilon}^{2}(t) d t \leq \int_{0}^{T} u^{\star 2}(t) d t, \forall \epsilon>0
$$

(10) and (12) show that

$$
\int_{0}^{T} u_{\epsilon}^{2}(t) d t \rightarrow \int_{0}^{T} u^{\star 2}(t) d t \text { as } \epsilon \rightarrow 0 .
$$

This result, joined to the weak convergence of $\left(u_{\epsilon}\right)_{\epsilon>0}$ towards $u^{\star}$ in $L^{2}[0, T]$, implies that:

$$
\lim _{\epsilon \rightarrow 0} \int_{0}^{T}\left(u_{\epsilon}(t)-u^{\star}(t)\right)^{2} d t=0 .
$$


Using (9), we obtain

$$
\int_{0}^{T} u^{\star 2}(t) d t \leq \int_{0}^{T} u^{2}(t) d t, \quad \forall u \in U_{a d}(\omega),
$$

hence, $u^{\star}$ is a solution of problem $(2)$.

Remark 1.6. (1) From the proof of theorem.1.5., one deduces that if the sequence $\left(u_{\epsilon}\right)_{\epsilon>0}$ is bounded in $L^{2}[0, T]$ then $U_{a d}(\omega) \neq \emptyset$.

(2) We do not give any result for the uniqueness except for the global case $(\omega=\Omega)$. We have the following result.

Proposition 1.7. Suppose that $U_{a d}(\Omega)$ is a nonempty convex set of $L^{2}[0, T]$ and $L^{2}(\Omega)$ has an orthonormal basis $\left(\phi_{n}\right)_{n}$ of eigenfunctions of $A$. In addition if A commutes with B, then problem (2) has only one solution.

Proof. First the existence of solution is ensured by theorem.1.5.

Without loss of generality, we may suppose that the eigenvalues of $A$ are simple. Now, $A$ and $B$ commute so, the mild solution of (1) can be written

$$
z_{u}(t)=S(t) e^{B \int_{0}^{t} u(s) d s} z_{0}
$$

where $\left(e^{B \int_{s}^{t} u(r) d r}\right)_{t \geq s}$ is the evolution operator generated by $u B$. For $z_{0} \in L^{2}(\Omega)$ we have

$$
z_{u}(t)=\sum_{n=1}^{+\infty} e^{\lambda_{n} t}\left\langle e^{B} \int_{0}^{t} u(s) d s z_{0}, \phi_{n}\right\rangle \phi_{n}
$$

Then

$$
z_{u}(T)-z_{d}=\sum_{n=1}^{+\infty}\left\langle e^{\lambda_{n} T} e^{B} \int_{0}^{T} u(s) d s\right.
$$

and

$$
\left\|z_{u}(T)-z_{d}\right\|^{2}=\sum_{n=1}^{+\infty}\left\langle e^{\lambda_{n} T} e^{B} \int_{0}^{T} u(s) d s\right.
$$

If $u, v$ are two distinct solutions of problem (2), then (13) implies that

$$
\int_{0}^{T} u(s) d s=\int_{0}^{T} v(s) d s
$$

The control $w=\frac{u+v}{2}$ lies in $U_{a d}(\Omega)\left(z_{w}(T)=S(T) e^{B} \int_{0}^{T} \frac{1}{2}[u(s)+v(s)] d s \quad z_{0}=z_{u}(T)\right)$, and

$$
\begin{aligned}
\|w\|_{L^{2}[0, T]}^{2} & =\frac{1}{4}\|u+v\|_{L^{2}[0, T]}^{2} \\
& <\frac{1}{2}\left[\|u\|_{L^{2}[0, T]}^{2}+\|v\|_{L^{2}[0, T]}^{2}\right]=\|u\|_{L^{2}[0, T]}^{2} .
\end{aligned}
$$

This contradiction implies that the minimum energy control is unique. 
Remark 1.8. (1) The above results remain true in the case of multi-controls, i.e., the system is described by

$$
\dot{z}(t)=A z(t)+\sum_{i=1}^{p} u_{i}(t) B_{i} z(t),
$$

where $\forall i, 1 \leq i \leq p, u_{i} \in L^{2}[0, T ; \mathbb{R}], B_{i}$ is a linear bounded operator on $Z$.

(2) We can solve in the same way the following general problem:

$$
\left\{\begin{array}{l}
\min \|u\|_{L^{2}[0, T]}^{2} \\
\text { with } \\
\left\langle\chi_{\omega} z_{u}(T)-z_{d}(T), G\left(\chi_{\omega} z_{u}(T)-z_{d}(T)\right)\right\rangle_{L^{2}(\omega)} \\
+\int_{0}^{T}\left\langle\chi_{\omega} z_{u}(t)-z_{d}(t), Q\left(\chi_{\omega} z_{u}(t)-z_{d}(t)\right)\right\rangle d t \\
\text { minimum }
\end{array}\right.
$$

where $z_{d}$ is a desired regular function.

The problem associated to (14) is

$$
\left\{\begin{array}{l}
\min \Phi_{\epsilon}(u) \\
u \in L^{2}[0, T]
\end{array}\right.
$$

with

$$
\begin{gathered}
\Phi_{\epsilon}(u)=\left\langle\chi_{\omega} z_{u}(T)-z_{d}(T), G\left(\chi_{\omega} z_{u}(T)-z_{d}(T)\right)\right\rangle_{L^{2}(\omega)}+\int_{0}^{T}\left[\left\langle\left(\chi_{\omega} z_{u}(t)-z_{d}(t), Q\left(\chi_{\omega} z_{u}(t)-z_{d}(t)\right)\right\rangle_{L^{2}(\omega)}\right.\right. \\
\left.+\epsilon u^{2}(t)\right] d t \epsilon>0,
\end{gathered}
$$

and its solution is given by

$$
u(t)=-\frac{1}{\epsilon}\left\langle B z(t), P(t) z(t)-U^{*}(T, t) \chi_{\omega}^{*} G z_{d}(T)-\int_{t}^{T} U^{*}(s, t) \chi_{\omega}^{*} Q z_{d}(s) d s\right\rangle .
$$

where $P$ is the self-adjoint and non negative operator solution of the equation:

$$
\left\{\begin{array}{l}
\frac{d}{d t}\langle P(t) y, z\rangle+\langle P(t) y,(A+u(t) B) z\rangle \\
+\langle(A+u(t) B) y, P(t) z\rangle+\left\langle\chi_{\omega}^{*} Q \chi_{\omega} y, z\right\rangle=0 \\
P(T)=\chi_{\omega}^{*} G \chi_{\omega} \text { where } y, z \in D(A) .
\end{array}\right.
$$

(3) If $z_{d}($.$) is exactly reachable with the control v$ then,

$$
\begin{aligned}
& u_{\epsilon} \rightarrow v \text { in } L^{2}[0, T] \text { as } \epsilon \rightarrow 0, \text { strongly, } \\
& \chi_{\omega} z_{\epsilon} \rightarrow z_{d} \text { in } C\left([0, T] ; L^{2}(\omega)\right) \text { strongly, }
\end{aligned}
$$

where, $u_{\epsilon}$ is a control which minimizes in $L^{2}[0, T]$ the quadratic cost:

$$
J_{\epsilon}(u)=\left\|\chi_{\omega} z_{u}(T)-z_{d}(T)\right\|_{L^{2}(\omega)}^{2}+\int_{0}^{T}\left[\left\langle\chi_{\omega} z_{u}(t)-z_{d}(t), \chi_{\omega} z_{u}(t)-z_{d}(t)\right\rangle_{L^{2}(\omega)}+\epsilon u^{2}(t)\right] d t(\epsilon>0) .
$$

We now deal with the case where $U_{a d}(\omega)$ is an empty set. 
Theorem 1.9. Suppose that $U_{a d}(\omega)$ is empty then

$$
\lim _{\epsilon \rightarrow 0}\left\|\chi_{\omega} z_{\epsilon}(T)-z_{d}\right\|_{L^{2}(\omega)}^{2}=\inf _{z \in R(T)}\left\|\chi_{\omega} z-z_{d}\right\|_{L^{2}(\omega)}^{2} .
$$

Proof.

$$
\text { Let } F=\left\{\left\|\chi_{\omega} z-z_{d}\right\|_{L^{2}(\omega)} \mid z \in R(T)\right\} \text {. }
$$

Then, $F$ is a nonempty subset of $\mathbb{R}^{+}$. Therefore, $F$ has a lower bound denoted $a$. According to proposition 1.1., $\left(J_{\epsilon}\left(u_{\epsilon}\right)\right)_{\epsilon>0}$ is a decreasing sequence as $\epsilon \rightarrow 0$, and $J_{\epsilon}\left(u_{\epsilon}\right) \geq 0, \quad \forall \epsilon>0$.

So, it converges in $\mathbb{R}$ towards a limit denoted $J$.

Similarly, $\left(\left\|\chi_{\omega} z_{\epsilon}(T)-z_{d}\right\|_{L^{2}(\omega)}\right)_{\epsilon>0}$ is a non negative and decreasing sequence. So, it converges in $\mathbb{R}$ towards a limit denoted $b$, as $\epsilon \rightarrow 0$.

Let us show that $b=a$ :

Suppose that $b>a$, then there exists $v \in L^{2}[0, T]$ such that:

$$
a<\left\|\chi_{\omega} z_{v}(T)-z_{d}\right\|_{L^{2}(\omega)}<b .
$$

Now,

$$
\begin{aligned}
& \left\|\chi_{\omega} z_{\epsilon}(T)-z_{d}\right\|_{L^{2}(\omega)}^{2}+\epsilon \int_{0}^{T} u_{\epsilon}^{2}(t) d t \\
& \leq\left\|\chi_{\omega} z_{v}(T)-z_{d}\right\|_{L^{2}(\omega)}^{2}+\epsilon \int_{0}^{T} v^{2}(t) d t .
\end{aligned}
$$

(16) and (17) imply that $\int_{0}^{T} u_{\epsilon}^{2}(t) \leq \int_{0}^{T} v^{2}(t) d t$.

Thus, according to remark 1.8, $U_{a d}(\omega)$ is nonempty, which is a contradiction.

Remark 1.10. (1) The Family of the control $\left(u_{\epsilon}\right)_{\epsilon>0}$ is not bounded in $L^{2}[0, T]$ (Remark 1.6.) and for a fixed $\epsilon$ and for all $\chi_{\omega} z_{v}(T)$ such that

$$
\left\|\chi_{\omega} z_{\epsilon}(T)-z_{d}\right\|_{L^{2}(\omega)}=\left\|\chi_{\omega} z_{v}(T)-z_{d}\right\|_{L^{2}(\omega)},
$$

according to (17) we have

$$
\int_{0}^{T} u_{\epsilon}^{2}(t) d t \leq \int_{0}^{T} v^{2}(t) d t .
$$

(2) The approach using to solve the optimal control problem assumes a bounded control operator, however the unbounded case may be carried out with similar manner taking more regular controls which allow regular system states. It means that the control is taken such that the state $z$ be in $Z=L^{2}(\Omega)$.

\subsection{Numerical approach and simulations}

We have seen that if an optimal control solution of the problem (2) exists, such control may be approximated by the $u_{\epsilon}$ solution of the problem (3) which in turn may be realized by the following formula

$$
\begin{cases}u_{n+1}(t) & =-n\left\langle B z_{n}(t), P_{n}(t) z_{n}(t)-U_{n}^{*}(T, t) \chi_{\omega}^{*} z_{d}\right\rangle \\ u_{0} & =0 .\end{cases}
$$

where $P_{n}$ is the self-adjoint and non negative operator solution of the Riccati equation

$$
\left\{\begin{array}{l}
\frac{d}{d t}\left\langle P_{n}(t) y, z\right\rangle+\left\langle P_{n}(t) y,\left(A+u_{n}(t) B\right) z\right\rangle \\
+\left\langle\left(A+u_{n}(t) B\right) y, P_{n}(t) z\right\rangle=0 \\
P_{n}(T)=\chi_{\omega}^{*} \chi_{\omega} \text { with } y, z \in D(A) .
\end{array}\right.
$$


which the solution can be achieved by the algorithm given in [6].

This allows to consider the following algorithm :

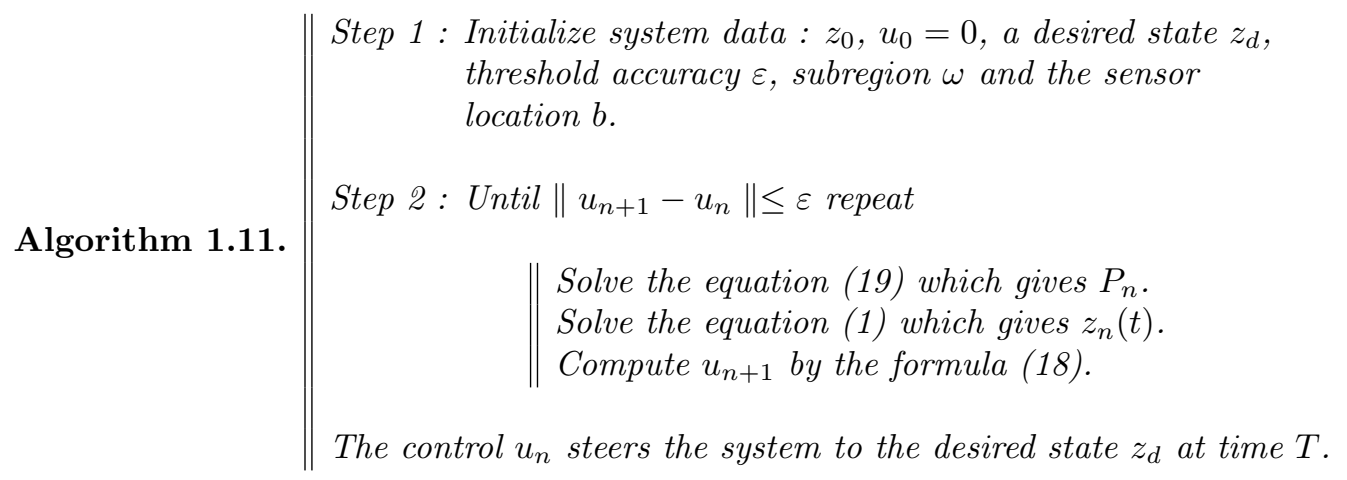

To illustrate the above algorithm, consider the following : examples.

Example 1.12. let $\Omega=] 0,1[$ and consider the bilinear system described by the following evolution equation:

$$
\left\{\begin{aligned}
\frac{\partial z}{\partial t}(x, t) & =\alpha \frac{\partial^{2} z(x, t)}{\partial x^{2}}+\beta z(x, t) & & \\
& +\gamma u(t) z(x, t) & & \text { in } \Omega \times] 0, T[ \\
z(x, 0) & =z_{0}(x) & & \text { in } \Omega \\
z(0, t) & =z(1, t)=0 & & \text { on }] 0, T[.
\end{aligned}\right.
$$

where $\alpha, \beta$ and $\gamma$ are positive constants.

This equation may represent a simplified model of the temperature distribution in a furnace.

The system (20) looks like (1) with $\tilde{A}=\alpha \frac{\partial^{2}}{\partial x^{2}}+\beta$ with domain

$$
D(\tilde{A})=\left\{z \in H^{2}(0,1) \mid z(0)=z(1)=0\right\} .
$$

The operator $\tilde{A}$ admits a set of eigenfunctions $\phi_{i}($.$) associated to the eigenvalues \lambda_{i}$ given by

$$
\phi_{i}(x)=\sqrt{2} \sin (i \pi x) ; \lambda_{i}=\beta-\alpha i^{2} \pi^{2}, i \geq 1 .
$$

The solution (20) is approximated by : $z(x, t) \simeq \sum_{i=1}^{M} a_{i}(t) \phi_{i}(x)$.

Let $z_{0}(x)=\sin (\pi x), z_{d}(x)=8 x(1-x), \alpha=0.01, \beta=0.01, \gamma=0.02, \varepsilon=0.0001$ and $T=1$. Augmenting the truncations order $M$ beyond 5 does not improve the simulation results.

Using the above algorithm for different region of $\omega$ and after 7 iterations we have 


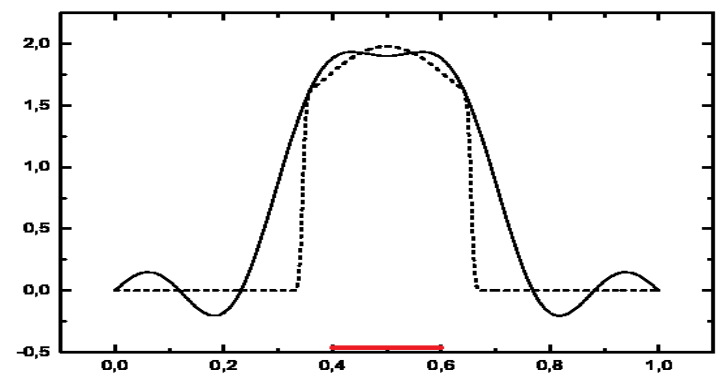

Figure 1. Desired (...) and final state $(-)$ on $\omega=] 0,4 ; 0,6[$.

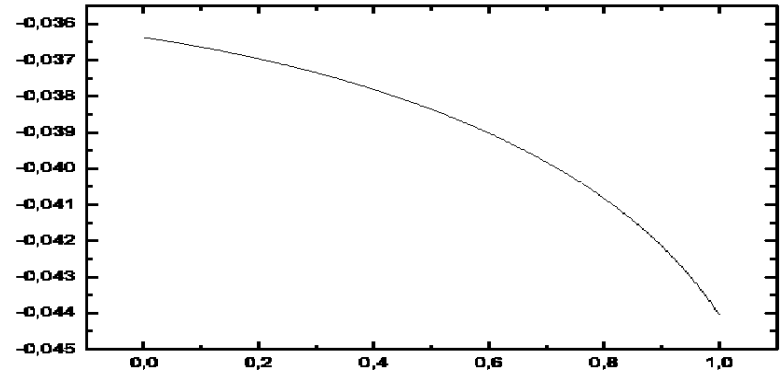

Figure 2. Evolution of the control function on $[0 ; 1]$.

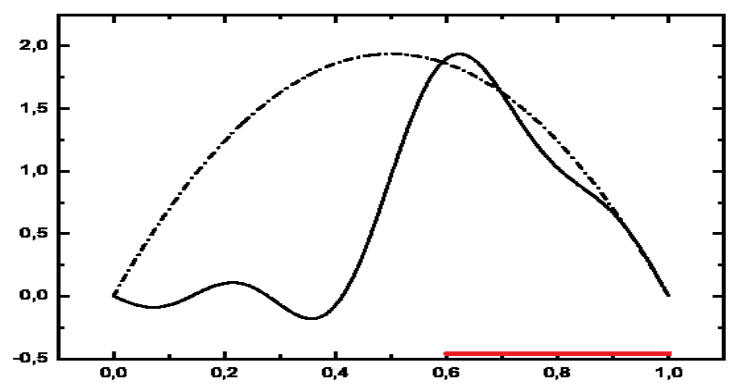

Figure 3. Desired $(\ldots)$ and final state $(-)$ on $\omega=] 0,6 ; 1[$.

Example 1.13. Let consider the bilinear system with domain $\Omega=] 0,1[$ and described by the following equation:

$$
\left\{\begin{array}{llr}
\frac{\partial z}{\partial t}(x, t) & =\alpha \frac{\partial^{2} z(x, t)}{\partial x^{2}}+\beta z(x, t)+ & \\
& +\delta(x-b) u(t) & \text { in } \Omega \times] 0, T[ \\
z(x, 0) & =z_{0}(x) & \text { in } \Omega \\
z(0, t) & =z(1, t)=0 & \text { on }] 0, T[
\end{array}\right.
$$




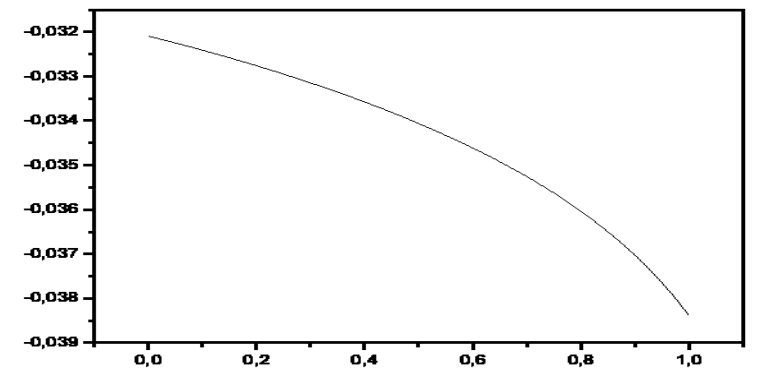

FiguRE 4. Evolution of the control function on $[0 ; 1]$.

$\tilde{A}=\alpha \frac{\partial^{2}}{\partial x^{2}}+\beta$ is the dynamical system with domain $D(\tilde{A})=\left\{z \in H^{2}(0,1) \mid z(0)=z(1)=0\right\}$ and $\delta$ is the Dirac function.

Let $z_{0}(x)=6.4 x(1-x), \alpha=0.01, \beta=0.01$, $\tilde{\gamma}=0.02, \varepsilon=10^{-4}, T=1$ and $b=0.1$. Applying the above algorithm, the simulation gives.

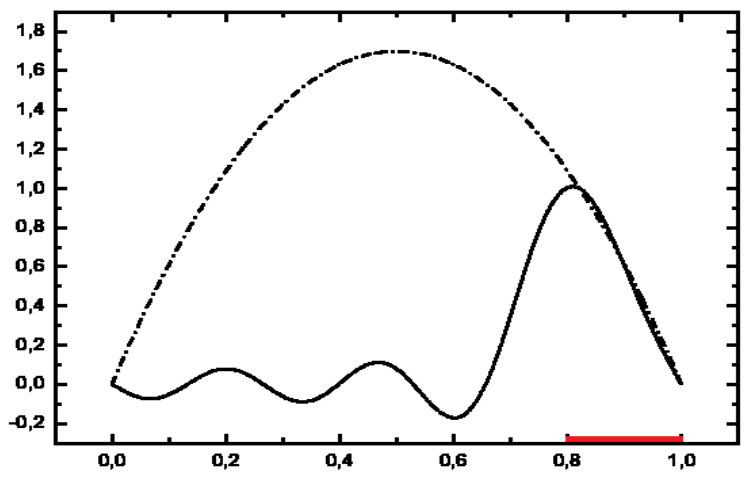

Figure 5. Desired (...) and final state $(-)$ on $\omega=] 0,8 ; 1[$.

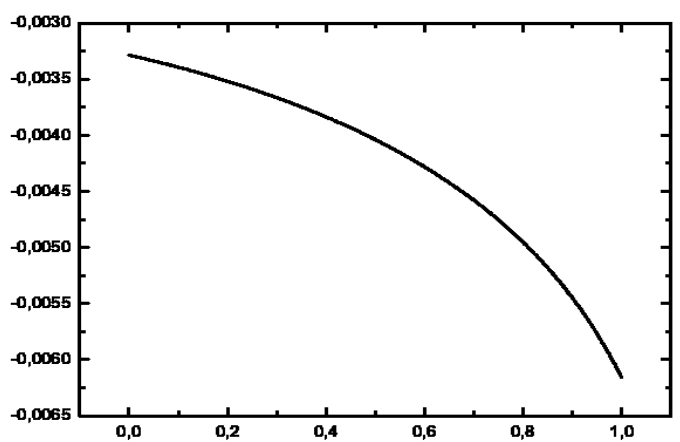

Figure 6. Evolution of the control function on $[0 ; 1]$. 


\section{Bounded CONTROL CASE}

let us consider again the bilinear system as given in (1) described by the equation

$$
\begin{cases}\frac{\partial z(x, t)}{\partial t}=A z(x, t)+u(t) B z(x, t) & \Pi \\ z(x, 0)=z^{0}(x) & \Omega \\ z(x, t)=0 & \Sigma\end{cases}
$$

where $A$ generates a $C^{0}$ semigroup $(S(t))_{t>0}$ of bounded linear operators on a $L^{2}(\Omega)$. $B: L^{2}(\Omega) \longrightarrow L^{2}(\Omega)$ is a linear bounded operator and the control $u(t) \in U_{M}$ where

$$
U_{M}=\left\{u \in L^{\infty}([0, T]) \mid-M \leq u(t) \leq M\right\} \text { and } \mathrm{M} \text { is a positif constant }
$$

The system $(22)$ is bilinear in the pair $(u, z)$, and its solution $z$ is a nonlinear function with respect to $u$. For a given $z^{0} \in L^{2}(\Omega),(22)$ is written as

$$
z_{u}(x, t)=S(t) z^{0}(x)+\int_{0}^{t} S(t-s) u(s) B z_{u}(x, s) d s .
$$

and solutions of (23) are called mild solutions of (22). The existence of a unique solution $z(x, t)$ in $\mathcal{C}\left([0, T] ; L^{2}(\Omega)\right)$ satisfying $(23)$, follows from standard results as in $[3]$.

Here the problem (2) is equivalent to the following

$$
\left\{\begin{array}{l}
\text { Minimizes }\|u\|_{L^{2}([0, T])}^{2} \\
u \in U_{M} \\
\text { Under the constraint: } \\
\left\|\chi_{\omega} z_{u}(T)-z^{d}\right\|_{L^{2}(\omega)}^{2} \text { is minimum }
\end{array}\right.
$$

Here we deal with the problem (24) with bounded controls, this case is the most natural, and often encountered in real applications.

To characterize the optimal control solution of (24), we propose also an approach based on quadratic cost control problem which involves the minimization of the norm control and the final state error.

We will discuss the quadratic cost control problem associated to (24). We show the existence of an optimal control by a minimizing sequence argument. And, we derive a characterization for optimal controls, using the solution of an optimality system, that consists of the equation (22) coupled with an adjoint equation. We solve the regional minimum control problem. and we give a numerical approach leading to an algorithm illustrated through numerical simulations.

\subsection{Regional quadratic bounded control problem}

Consider the regional quadratic control problem

$$
\left\{\begin{array}{l}
\min J(u) \\
u \in U_{M}
\end{array}\right.
$$

where $J$ is the functional

$$
J(u)=\left\|\chi_{\omega} z_{u}(T)-z^{d}\right\|_{L^{2}(\omega)}^{2}+\frac{\epsilon}{2}\|u\|_{L^{2}([0, T])}^{2}
$$

$\epsilon>0$ is a positive constant.

The goal of this section is to justify that this minimum can be achieved, and characterize an optimal control $u^{*} \in U_{M}$ solution of $(25)$. 
Theorem 2.1. There exists a pair $\left(\bar{z}, u^{*}\right) \in \mathcal{C}\left([0, T] ; L^{2}(\Omega)\right) \times U_{M}$, such that $\bar{z}$ is the unique solution of

$$
\begin{cases}\frac{\partial z(x, t)}{\partial t}=A z(x, t)+u^{*}(t) B z(x, t) & \Pi \\ z(x, 0)=z^{0}(x) & \Omega \\ z(x, t)=0 & \Sigma\end{cases}
$$

and $u^{*}$ is an optimal control which minimizes the objective functional $J(u)$ over $U_{M}$.

Proof. The solutions $z(x, t)$ of the equation (22) are weak solutions in $W=L^{2}\left(0, T ; L^{2}(\Omega)\right)($ see $[2,4])$.

Using (23), and the bound $C$ of the strongly continuous semigroup $(S(t))_{t \geq 0}$ in the interval $[0, T]$ (see [5]), we have

$$
\left\|z_{u}(t)\right\|_{W} \leq C\left\|z^{0}\right\|_{L^{2}(\Omega)}+C\|B\| \int_{0}^{t}|u(s)|\left\|z_{u}(s)\right\|_{W} d s
$$

Using Gronwall inequality, we obtain

$$
\left\|z_{u}(t)\right\|_{W} \leq C_{1} \exp (C\|B\| M T)
$$

with $C_{1}=C\left\|z^{0}\right\|_{L^{2}(\Omega)}$.

The set $\left\{J(u) \mid u \in U_{M}\right\}$ is nonempty and bounded from below.

Let the minimizing sequence $\left\{u_{n}\right\}, n=1,2 \ldots$, be such that

$$
J^{*}=\lim _{n \rightarrow+\infty} J\left(u_{n}\right)=\inf _{u \in U_{M}} J(u)
$$

$J\left(u_{n}\right)$ is then bounded, it follows that $\left\|u_{n}\right\|_{L^{2}([0, T])}$ is also bounded.

Let $z_{n}(x, t)=z_{u_{n}}(x, t)$, we conclude from (28), that $\left\|z_{n}\right\|_{W}$ are uniformly bounded independently of $n$.

From the priori estimates we deduce that

$$
\left\|A z_{n}\right\|_{W} \leq M_{1},\left\|u_{n} B z_{n}\right\|_{W} \leq M_{2}, \text { and }\left\|z_{n}^{\prime}\right\|_{W} \leq M_{3}
$$

where $M_{i},\{i=1,2,3\}$ are positive constants.

From these bounds, there exist subsequences with the following convergence properties:

$$
\begin{array}{lll}
u_{n} \rightarrow u^{*} & \text { weakly in } & L^{2}(0, T) \\
z_{n} \rightarrow \bar{z} & \text { weakly in } & W \\
A z_{n} \rightarrow \chi & \text { weakly in } & W \\
u_{n} B\left(z_{n}\right) \rightarrow \Lambda & \text { weakly in } & W \\
z_{n}^{\prime} \rightarrow \Psi & \text { weakly in } & W
\end{array}
$$

By classical argument, (see [25]), we verify that $\bar{z}(0)=z^{0}$, and we can pass to the limit in equation (22), associated with $\left(u_{n}, z_{u_{n}}\right)$ as $n \longrightarrow \infty$. Then $\bar{z}^{\prime}=\Psi, A \bar{z}=\chi$ and $u^{*} B \bar{z}=\Lambda$. Then $\bar{z}=z\left(u^{*}\right)$.

Now we verify that $u^{*}$ is an optimal control.

Using the lower semi continuity of the norms and applying Fatou's Lemma, we deduce

$$
\begin{aligned}
J\left(u^{*}\right) & =\inf _{n} \int_{\omega}\left(\chi_{\omega} z_{n}(x, T)-z^{d}\right)^{2}+\frac{\epsilon}{2} \int_{0}^{T} u_{n}^{2}(t) d t \\
& \leq \lim _{n \longrightarrow \infty} J\left(u_{n}\right)=\inf _{u} J(u)
\end{aligned}
$$

Then $u^{*}$ is an optimal control. 
Remark 2.2. If we consider the state equation with a source term $f \in L^{\infty}\left(0, T ; L^{2}(\Omega)\right)$,

$$
\frac{\partial z}{\partial t}=A z+u(t) B z+f \Pi
$$

the same well-posedness and regularity results as (22) hold, but the constant $C_{1}$ in (28) takes the form

$$
C_{1}=C\left(\left\|z^{0}\right\|_{L^{2}(\Omega)}+\|f\|_{L^{\infty}\left(0, T ; L^{2}(\Omega)\right)}\right) .
$$

To obtain a characterization of an optimal control, we must derive the optimality system by differentiating the cost functional $J(u)$ with respect to the control $u$.

We examine the differentiability of $u \longrightarrow z(u)$ with respect to $\mathrm{u}$.

Lemma 2.3. The map $u \in U_{M} \longrightarrow z=z(u) \in W$ is differentiable in the following sense:

$$
\frac{z(u+\rho h)-z(u)}{\rho} \rightarrow \psi \text { weakly in } W
$$

as $\rho \longrightarrow 0$ where $u+\rho h \in U_{M}, h \in L^{\infty}([0, T])$. Moreover $\psi=\psi(z, h)$ satisfies

$$
\begin{cases}\psi_{t}(x, t)=A \psi(x, t)+u(t) B \psi(x, t)+h(t) B \bar{z}(x, t) & \Pi \\ \psi(x, 0)=\psi_{0}(x)=0 & \Omega \\ \psi(x, t)=0 & \Sigma\end{cases}
$$

where $\bar{z}=z\left(u^{*}\right)$.

Proof. Consider $z_{\rho}=z(u+\rho h)$, then $\varphi(x, t)=\frac{z_{\rho}-z}{\rho}(x, t)$ is a weak solution of

$$
\begin{cases}\frac{\partial \varphi(x, t)}{\partial t}=A \varphi(x, t)+u(t) B \varphi(x, t)+h(t) B z_{\rho} & \Pi \\ \varphi(x, 0)=0 & \Omega \\ \varphi(x, t)=0 & \Sigma\end{cases}
$$

Using (28) and Remark 2.2., we obtain

$$
\begin{aligned}
\left\|\frac{z_{\rho}-z}{\rho}\right\|_{W} & \leq\left\|h B z_{\rho}\right\|_{L^{\infty}\left(0, T ; L^{2}(\Omega)\right)} \exp (C M T\|B\|) \\
& \leq C_{2}
\end{aligned}
$$

where $C_{2}$ is independent of $\rho$ since the bound on $\left\|z_{\rho}\right\|_{L^{\infty}\left(0, T ; L^{2}(\Omega)\right)}$ is independent of $\rho$, and the weak convergence to $\psi$ is obtained. We also have (see [10])

$$
\frac{\left(z_{\rho}-z\right)}{\rho} \longrightarrow \text { strongly in } L^{2}(\Omega) \text { as } \rho \longrightarrow 0 .
$$

We conclude that $\psi$ satisfies (31).

We have also the weak convergence of the traces $\frac{\left(z_{\rho}-z\right)}{\rho}(x, T)$, in $L^{2}(\Omega)$ (see [10]).

We are ready to characterize the optimal control, by deriving the optimality system through differentiating $J(u)$ with respect to $u$ at an optimal control. 
Theorem 2.4. Given an optimal control $u_{\epsilon}$ in $U_{M}$, and the corresponding solution $\bar{z}=z\left(u_{\epsilon}\right)$ to (22), the adjoint equation

$$
\begin{cases}\frac{\partial p(x, t)}{\partial t}=-A^{*} p(x, t)-u_{\epsilon}(t) B^{*} p(x, t) & \Pi \\ p(x, T)=\left(\bar{z}(T)-\chi_{\omega}^{*} z^{d}\right) & \Omega \\ p(x, t)=0 & \Sigma\end{cases}
$$

has a unique solution $p \in W$.

The operator $A^{*}$, the adjoint operator of the operator $A$, generates a strongly continuous semigroup $\left(S^{*}(t)\right)_{t \geq 0}$ of bounded linear operators on $L^{2}(\Omega)$, and $B^{*}$ is the linear bounded operator adjoint of the operator $B, \chi_{\omega}^{*}$ is the adjoint operator of $\chi_{\omega}$, defined from $L^{2}(\omega) \longrightarrow L^{2}(\Omega)$, and given by

$$
\chi_{\omega}^{*} z(x)=\left\{\begin{array}{cc}
z(x) & x \in \omega \\
0 & x \in \Omega \backslash \omega
\end{array}\right.
$$

Moreover,

$$
u_{\epsilon}(t)=\max \left(-M, \min \left(-\frac{2}{\epsilon} \int_{\omega} p B \bar{z} d x, M\right)\right)
$$

Proof. Consider $u \in U_{M}$ and let $h \in L^{\infty}(0, T)$ such that $u+\rho h \in U_{M}$ for $\rho>0$. The derivative of $J(u)$ with respect to $u$ in the direction $h$ satisfies.

$$
\begin{aligned}
0 & \leq \lim _{\rho \longrightarrow 0} \frac{J(u+\rho h)-J(u)}{\rho} \\
& =\lim _{\rho \longrightarrow 0} \int_{\omega} \frac{\left(\chi_{\omega} z_{\rho}-z^{d}\right)^{2}-\left(\chi_{\omega} z-z^{d}\right)^{2}}{\rho} d x \\
& +\lim _{\rho \longrightarrow 0} \frac{\epsilon}{2} \int_{0}^{T} \frac{(u+\rho h)^{2}-u^{2}}{\rho}(t) d t \\
& =\lim _{\rho \longrightarrow 0} \int_{\omega} \chi_{\omega} \frac{\left(z_{\rho}-z\right)}{\rho}\left(\chi_{\omega} z_{\rho}+\chi_{\omega} z-2 z^{d}\right) d x \\
& +\lim _{\rho \longrightarrow 0} \frac{\epsilon}{2} \int_{0}^{T}\left(2 h u+\rho h^{2}\right) d t \\
& =2 \int_{\omega} \chi_{\omega} \psi(x, T) \chi_{\omega}\left(z(x, T)-\chi_{\omega}^{*} z^{d}\right) d x \\
& +\epsilon \int_{0}^{T} h z d t \\
& =2 \int_{\omega} \chi_{\omega} \psi(x, T) \chi_{\omega} p(x, T) d x+\epsilon \int_{0}^{T} h u d t \\
& =2 \int_{\omega} \chi_{\omega}^{*} \chi_{\omega}\left[\int_{0}^{T} \frac{\partial p}{\partial t} \psi d t+\int_{0}^{T} p \frac{\partial \psi}{\partial t} d t\right] d x \\
& +\epsilon \int_{0}^{T} h u d t
\end{aligned}
$$

Integrating by parts, yields

$$
\begin{aligned}
0 & \leq \lim _{\rho \longrightarrow 0} \frac{J(u+\rho h)-J(u)}{\rho} \\
& =2 \int_{\omega} \chi_{\omega} \chi_{\omega}\left[\int_{0}^{T} \psi \frac{\partial p}{\partial t} d t+\int_{0}^{T} \frac{\partial \psi}{\partial t} p d t\right] d x \epsilon \int_{0}^{T} h u d t
\end{aligned}
$$


The system (31) gives

$$
\begin{aligned}
0 & \leq \frac{1}{2} \lim _{\rho \longrightarrow 0} \frac{J(u+\rho h)-J(u)}{\rho} \\
& =\int_{\omega} \chi_{\omega}^{*} \chi_{\omega} \int_{0}^{T} \psi \frac{\partial p}{\partial t} d t d x+\epsilon \int_{0}^{T} h u d t \\
& +\int_{\omega} \chi_{\omega}^{*} \chi_{\omega}\left[\int_{0}^{T}(A \psi+u(t) B \psi+h(t) B \bar{z}) p d t\right] d x
\end{aligned}
$$

And using the system (33) we obtain

$$
\begin{aligned}
0 & \leq \lim _{\rho \longrightarrow 0} \frac{J\left(u_{\epsilon}(t)+\rho h\right)-J\left(u_{\epsilon}(t)\right)}{\rho} \\
& \leq 2 \int_{\omega} \chi_{\omega}^{*} \chi_{\omega}\left[\int_{0}^{T} \psi\left(\frac{\partial p}{\partial t}+A^{*} p+u_{\epsilon}(t) B^{*} p\right) d t\right] d x \\
& +2 \int_{\omega} \chi_{\omega}^{*} \chi_{\omega} \int_{0}^{T} h(t) B \bar{z} p d t d x+\epsilon \int_{0}^{T} h u_{\epsilon}(t) d t \\
& =2 \int_{0}^{T} h(t)\left\langle\chi_{\omega} B \bar{z} ; \chi_{\omega} p\right\rangle_{L^{2}(\omega)}+\epsilon \int_{0}^{T} h(t) u_{\epsilon}(t) d t \\
& =\int_{0}^{T}\left[2\left\langle\chi_{\omega} B \bar{z}(x, t) ; \chi_{\omega} p(t)\right\rangle_{L^{2}(\omega)}+\epsilon u_{\epsilon}(t)\right] h(t) d t
\end{aligned}
$$

Thus, using the arbitrary variation of $h$, and bounds on the control set $U_{M}$, we have

$$
u_{\epsilon}(t)=\max \left(-M, \min \left(-\frac{2}{\epsilon}\left\langle\chi_{\omega} B \bar{z} ; \chi_{\omega} p\right\rangle_{L^{2}(\omega)}, M\right)\right)
$$

\subsection{Regional minimum energy bounded control problem}

Next, let us consider the problem (24), the set of admissible controls

$$
U_{a d}(\omega)=\left\{u \in U_{M} / \quad\left\|\chi_{\omega} z_{u}(T)-z^{d}\right\|_{L^{2}(\omega)} \text { is minimum }\right\}
$$

and the set of reachable states at time $T$ from $z^{0}, R_{M}(T)=\bigcup_{u \in U_{M}}\left\{z_{u}(T)\right\}$;

Proposition 2.5. (1) The sequence $\left(J_{\epsilon}\left(u_{\epsilon}\right)\right)_{\epsilon>0}$ is increasing with respect to $\epsilon$.

(2) The sequence $\left(\int_{0}^{T} u_{\epsilon}^{2}(t) d t\right)_{\epsilon>0}$ is decreasing with respect to $\epsilon$.

(3) The sequence $\left(\left\|\chi_{\omega} z_{\epsilon}(T)-z^{d}\right\|_{L^{2}(\omega)}^{2}\right)_{\epsilon>0}$ is increasing with respect to $\epsilon$. and $\forall \epsilon>0$

$$
\left\|\chi_{\omega} z_{\epsilon}(T)-z^{d}\right\|_{L^{2}(\omega)} \leq\left\|\chi_{\omega} S(T) z^{0}-z^{d}\right\|_{L^{2}(\omega)}
$$

In particular, $\left(\chi_{\omega} z_{\epsilon}(T)-z^{d}\right)_{\epsilon>0}$ converges weakly in $L^{2}(\omega)$. 
Proof. Let $0<\epsilon_{1}<\epsilon_{2}$, using consecutively the optimality of $u_{\epsilon_{1}}$ for $J_{\epsilon_{1}}$ and the optimality of $u_{\epsilon_{2}}$ for $J_{\epsilon_{2}}$, we have

This implies that :

$$
\begin{aligned}
J_{\epsilon_{1}}\left(u_{\epsilon_{1}}\right) & =\left\|\chi_{\omega} z_{\epsilon_{1}}(T)-z^{d}\right\|_{L^{2}(\omega)}^{2}+\frac{\epsilon_{1}}{2} \int_{0}^{T} u_{\epsilon_{1}}^{2}(t) d t \\
& \leq\left\|\chi_{\omega} z_{\epsilon_{2}}(T)-z^{d}\right\|_{L^{2}(\omega)}^{2}+\frac{\epsilon_{1}}{2} \int_{0}^{T} u_{\epsilon_{2}}^{2}(t) d t \\
& \leq\left\|\chi_{\omega} z_{\epsilon_{2}}(T)-z^{d}\right\|_{L^{2}(\omega)}^{2}+\frac{\epsilon_{2}}{2} \int_{0}^{T} u_{\epsilon_{2}}^{2}(t) d t \\
& \leq\left\|\chi_{\omega} z_{\epsilon_{1}}(T)-z^{d}\right\|_{L^{2}(\omega)}^{2}+\frac{\epsilon_{2}}{2} \int_{0}^{T} u_{\epsilon_{1}}^{2}(t) d t
\end{aligned}
$$

$$
J_{\epsilon_{1}}\left(u_{\epsilon_{1}}\right) \leq J_{\epsilon_{2}}\left(u_{\epsilon_{2}}\right)
$$

From (40), we obtain $J_{\epsilon_{2}}\left(u_{\epsilon_{2}}\right)-J_{\epsilon_{1}}\left(u_{\epsilon_{2}}\right) \leq J_{\epsilon_{2}}\left(u_{\epsilon_{1}}\right)-J_{\epsilon_{1}}\left(u_{\eta \epsilon_{1}}\right)$

which gives $\int_{0}^{T} u_{\epsilon_{2}}^{2}(t) d t \leq \int_{0}^{T} u_{\epsilon_{1}}^{2}(t) d t$ and so $\left\|\chi_{\omega} z_{\epsilon_{1}}(T)-z^{d}\right\|_{L^{2}(\omega)}^{2} \leq\left\|\chi_{\omega} z_{\epsilon_{2}}(T)-z^{d}\right\|_{L^{2}(\omega)}^{2}$, which shows statements 1., 2. and the first part of 3 .

Now, for $u=0$, we have $z(T)=S(T) z^{0}$, and $\forall \epsilon>0$

$$
\left\|\chi_{\omega} z_{\epsilon}(T)-z^{d}\right\|_{L^{2}(\omega)}^{2}+\epsilon \int_{0}^{T} u_{\epsilon}^{2}(t) d t \leq\left\|\chi_{\omega} S(T) z^{0}-z^{d}\right\|_{L^{2}(\omega)}^{2}
$$

Then, $\forall \epsilon>0 \quad 0 \leq\left\|\chi_{\omega} z_{\epsilon}(T)-z^{d}\right\|_{L^{2}(\omega)}^{2} \leq\left\|\chi_{\omega} S(t) z^{0}-z^{d}\right\|_{L^{2}(\omega)}^{2}$.

Finally, $\left(\left\|\chi_{\omega} z_{\epsilon}(T)-z^{d}\right\|_{L^{2}(\omega)}\right)_{\epsilon>0}$ is bounded. Then $\left(\chi_{\omega} z_{\epsilon}(T)-z^{d}\right)_{\epsilon>0}$ converges weakly in $L^{2}(\omega)$.

And we have the main result.

Theorem 2.6. Let $u_{\epsilon}$ be a solution of (25) and assume that $U_{a d}(\omega)$ is nonempty, then $u_{\epsilon} \rightarrow \bar{u}$ as $\epsilon \rightarrow$ 0 in $L^{2}([0, T])$ and $\chi_{\omega} z_{\epsilon} \rightarrow \chi_{\omega} z_{\bar{u}}$ in $C\left([0, T] ; L^{2}(\omega)\right)$. Moreover $\bar{u} \in U_{M}$ is a solution of the problem (24).

Proof. Using the optimality of $u_{\epsilon}$ for $J_{\epsilon}$ we have, $\forall \epsilon>0, u \in U_{M} J_{\epsilon}\left(u_{\eta}\right) \leq J_{\eta}(u)$, i.e.,

$$
\left\|\chi_{\omega} z_{\epsilon}(T)-z^{d}\right\|_{L^{2}(\omega)}^{2}+\frac{\epsilon}{2} \int_{0}^{T} u_{\epsilon}^{2}(t) d t \leq\left\|\chi_{\omega} z_{u}(T)-z^{d}\right\|_{L^{2}(\omega)}^{2}+\frac{\epsilon}{2} \int_{0}^{T} u^{2}(t) d t .
$$

Since $U_{a d}(\omega)$ is nonempty, there exists $v \in U_{M}$, such that

$$
\left\|\chi_{\omega} z_{v}(T)-z^{d}\right\|_{L^{2}(\omega)}^{2}=\min _{u \in R_{M}(T)}\left\|\chi_{\omega} z-z^{d}\right\|_{L^{2}(\omega)}^{2}
$$

So, we have

$$
\forall u \in U_{a d}(\omega) \quad \int_{0}^{T} u_{\epsilon}^{2}(t) d t \leq \int_{0}^{T} u^{2}(t) d t, \quad \forall \epsilon>0
$$

Therefore, we can extract a subsequence also denoted $\left(u_{\epsilon}\right)_{\epsilon>0}$ such that $u_{\epsilon} \rightarrow \bar{u}$ weakly in $L^{2}([0, T])$, and $z_{\epsilon} \rightarrow z_{\bar{u}}$ strongly in $C\left([0, T] ; L^{2}(\Omega)\right)$ as $\epsilon \rightarrow 0$, (see [3]), and this implies $\chi_{\omega} z_{\epsilon} \rightarrow \chi_{\omega} z_{\bar{u}}$ strongly in $C\left([0, T] ; L^{2}(\omega)\right.$ ). Furthermore,

$$
\begin{aligned}
& \liminf _{\epsilon} \int_{0}^{T} u_{\epsilon}^{2}(t) d t \geq \int_{0}^{T} \bar{u}^{2}(t) d t \\
& \text { and } \liminf _{\epsilon} J_{\epsilon}\left(u_{\epsilon}\right) \geq\left\|\chi_{\omega} z_{\bar{u}}(T)-z^{d}\right\|_{L^{2}(\omega)}^{2}
\end{aligned}
$$


Moreover, $J_{\epsilon}\left(u_{\epsilon}\right) \leq J_{\epsilon}(u) \forall u \in U_{M}$ so, $\lim _{\epsilon} \sup J_{\epsilon}\left(u_{\epsilon}\right) \leq\left\|\chi_{\omega} z_{u}(T)-z^{d}\right\|_{L^{2}(\omega)}^{2}, \forall u \in U_{M}$ and in particular,

$$
\begin{aligned}
\limsup _{\epsilon} J_{\epsilon}\left(u_{\epsilon}\right) & \leq\left\|\chi_{\omega} z_{v}(T)-z^{d}\right\|_{L^{2}(\omega)}^{2} \\
& \leq\left\|\chi_{\omega} z_{\bar{u}}(T)-z^{d}\right\|_{L^{2}(\omega)}^{2} \\
& \leq \liminf J_{\epsilon}\left(u_{\epsilon}\right) .
\end{aligned}
$$

Hence,

$$
\begin{aligned}
\lim _{\epsilon \rightarrow 0} J_{\epsilon}\left(u_{\epsilon}\right) & =\lim _{\epsilon \rightarrow 0}\left\|\chi_{\omega} z_{\epsilon}(T)-z^{d}\right\|_{L^{2}(\omega)}^{2} \\
& =\left\|\chi_{\omega} z_{\bar{u}}(T)-z^{d}\right\|_{L^{2}(\omega)}^{2} \\
& =\left\|\chi_{\omega} z_{v}(T)-z^{d}\right\|_{L^{2}(\omega)}^{2}
\end{aligned}
$$

Thus $\lim _{\epsilon \rightarrow 0}\left\|\chi_{\omega} z_{\epsilon}(T)-z^{d}\right\|_{L^{2}(\omega)}^{2}=\min _{z \in R(T)}\left\|\chi_{\omega} z-z^{d}\right\|_{L^{2}(\omega)}^{2}$ and $\bar{u} \in U_{a d}(\omega)$.

Furthermore,

$$
\left\|\chi_{\omega} z_{\epsilon}(T)-z^{d}\right\|_{L^{2}(\omega)}^{2}+\epsilon \int_{0}^{T} u_{\epsilon}^{2}(t) d t \leq\left\|\chi_{\omega} z_{\bar{u}}(T)-z^{d}\right\|_{L^{2}(\omega)}^{2}+\epsilon \int_{0}^{T} \bar{u}^{2}(t) d t .
$$

(44) gives

(43) and (45) show that

$$
\int_{0}^{T} u_{\epsilon}^{2}(t) d t \leq \int_{0}^{T} \bar{u}^{2}(t) d t \forall \epsilon>0
$$

$$
\int_{0}^{T} u_{\epsilon}^{2}(t) d t \rightarrow \int_{0}^{T} \bar{u}^{2}(t) d t \text { as } \epsilon \rightarrow 0
$$

This result, joined to the weak convergence of $\left(u_{\epsilon}\right)_{\epsilon>0}$ towards $\bar{u}$ in $L^{2}([0, T])$, implies that:

$$
\lim _{\epsilon \rightarrow 0} \int_{0}^{T}\left(u_{\epsilon}(t)-\bar{u}(t)\right)^{2} d t=0
$$

Using (42), we obtain $\int_{0}^{T} \bar{u}^{2}(t) d t \leq \int_{0}^{T} u^{2}(t) d t \forall u \in U_{a d}(\omega)$, so $\bar{u}$ is a solution of (24).

Remark 2.7. (1) It is easy to show that if $\left(u_{\epsilon}\right)_{\epsilon>0}$ converges strongly in $L^{\infty}(0, T)$ as $\epsilon \rightarrow 0$, then $U_{a d}(\omega) \neq$ $\emptyset$.

(2) The optimal control of the problem (25) is unique, when $T$ is sufficiently small, see [12].

(3) We haven't any result on the uniqueness of the optimal control of the problem (24), except for the global case (when $\omega=\Omega$ ), (see [27]).

Theorem 2.8. Suppose that $U_{a d}(\omega)$ is empty then

$$
\lim _{\epsilon \rightarrow 0}\left\|\chi_{\omega} z_{\epsilon}(T)-z^{d}\right\|_{L^{2}(\omega)}^{2}=\inf _{z \in R(T)}\left\|\chi_{\omega} z-z^{d}\right\|_{L^{2}(\omega)}^{2}
$$

Proof. The set $F=\left\{\left\|\chi_{\omega} z-z^{d}\right\|_{L^{2}(\omega)} \mid z \in R(T)\right\}$ is a nonempty subset of $\mathbb{R}^{+}$and has a lower bound denoted a. According to Proposition 2.5., $\left(J_{\epsilon}\left(u_{\epsilon}\right)\right)_{\epsilon>0}$ is a decreasing sequence as $\epsilon \rightarrow 0$, and $J_{\epsilon}\left(u_{\epsilon}\right) \geq 0, \quad \forall \epsilon>0$. So, it converges in $\mathbb{R}$ towards a limit denoted $J$.

Similarly, $\left(\left\|\chi_{\omega} z_{\epsilon}(T)-z^{d}\right\|_{L^{2}(\omega)}\right)_{\epsilon>0}$ is a non negative and decreasing sequence. So, it converges in $\mathbb{R}$ towards a limit denoted $b$, as $\epsilon \rightarrow 0$.

Let us show that $b=a$ :

Suppose that $b>a$, then there exists $v \in U_{M}$ such that

$$
a<\left\|\chi_{\omega} z_{v}(T)-z^{d}\right\|_{L^{2}(\omega)}<b
$$


Now,

$$
\left\|\chi_{\omega} z_{\epsilon}(T)-z^{d}\right\|_{L^{2}(\omega)}^{2}+\frac{\epsilon}{2} \int_{0}^{T} u_{\epsilon}^{2}(t) d t \leq\left\|\chi_{\omega} z_{v}(T)-z^{d}\right\|_{L^{2}(\omega)}^{2}+\frac{\epsilon}{2} \int_{0}^{T} v^{2}(t) d t
$$

(46) and (47) imply that $\int_{0}^{T} u_{\epsilon}^{2}(t) \leq \int_{0}^{T} v^{2}(t) d t$

Thus, according to Remark 2.7., $U_{a d}(\omega)$ is nonempty, which is a contradiction.

\subsection{Numerical approach}

Consider the one dimensional system

$$
\begin{cases}\frac{\partial z(x, t)}{\partial t}=\lambda \frac{\partial^{2} z(x, t)}{\partial x^{2}}-u(t) \beta z(x, t) & ] 0,1[\times] 0, T[ \\ z(0)=z^{0} & ] 0,1[ \\ z(0, t)=z(1, t)=0 & ] 0, T[\end{cases}
$$

and the problem (25), which solution $u_{\epsilon}$ is given by the formula

$$
u_{\epsilon}(t)=\max \left(-M, \min \left(\frac{2 \beta}{\epsilon}\left\langle\chi_{\omega} \bar{z} ; \chi_{\omega} p\right\rangle_{L^{2}(\omega)}, M\right)\right)
$$

where $\bar{z}=z\left(u_{\epsilon}\right)$ solution of (48), $p$ is solution of the adjoint equation associated to (48), and given by

$$
\begin{cases}-\frac{\partial p(x, t)}{\partial t}=\lambda \frac{\partial^{2} p(x, t)}{\partial x^{2}}-u(t) \beta p(x, t) & ] 0,1[\times] 0, T[ \\ p(T)=\left(\bar{z}(T)-\chi_{\omega}^{*} z^{d}\right), & ] 0,1[ \\ p(0, t)=p(1, t)=0 & ] 0, T[\end{cases}
$$

The following result enables us to simplify the above expression of $u_{\epsilon}(t)$.

Proposition 2.9. Consider the system (48), for M large enough, the optimal control is given by

$$
u_{\epsilon}(t)=\frac{2 \beta}{\epsilon}\left\langle\chi_{\omega} \bar{z}(x, t) ; \chi_{\omega} p(x, t)\right\rangle_{L^{2}(\omega)}
$$

Proof. Since $u=0 \in U_{M}$, we have,

$$
\begin{aligned}
J(0)=\left\|\chi_{\omega} z_{0}(T)-z^{d}\right\|_{L^{2}(\omega)}^{2} & \geq J\left(u_{\epsilon}\right) \\
& \geq \frac{\epsilon}{2}\left\|u_{\epsilon}(t)\right\|_{L^{2}(0, T)}^{2}
\end{aligned}
$$

where $z_{0}$ satisfies (48) with control $u=0$ and $u_{\epsilon} \in U_{M}$ is an optimal control. Thus $J(0)$ gives a bound of $\left\|u_{\epsilon}(t)\right\|_{L^{2}(0, T)}^{2}$ that is independent of M.

On an interval $(0, \tau)$ with $\tau \in[0, T], z=z\left(u_{\epsilon}\right)$ the solution of (48) satisfy

$$
\int_{0}^{1} \int_{0}^{\tau} \frac{\partial z(x, t)}{\partial t} z(x, t) d t d x=\lambda \int_{0}^{1} \int_{0}^{\tau} \frac{\partial^{2} z}{\partial x^{2}} z d t d x-u_{\epsilon}(t) \beta z^{2}(x, t) d t d x
$$


So

$$
\int_{0}^{1}\left[\frac{z^{2}(x, t)}{2}\right]_{0}^{\tau} d x=-\lambda \int_{0}^{\tau} \int_{0}^{1}\left(\frac{\partial z(x, t)}{\partial x}\right)^{2} d x d t-\int_{0}^{1} \int_{0}^{\tau} u_{\epsilon}(t) \beta z^{2}(x, t) d t d x
$$

which gives

$$
\begin{aligned}
\frac{1}{2} \int_{0}^{1} z^{2}(x, \tau) d x & =\frac{1}{2} \int_{0}^{1}\left(z^{0}\right)^{2}(x) d x \\
& +\frac{\beta}{2} \int_{0}^{1} \int_{0}^{\tau} u_{\epsilon}^{2}(t) z^{2}(x, t) d t d x \\
& +\frac{\beta}{2} \int_{0}^{1} \int_{0}^{\tau} z^{2}(x, t) d t d x
\end{aligned}
$$

Rearranging yields

$$
\int_{0}^{1} z^{2}(x, \tau) d x=\int_{0}^{1}\left(z^{0}\right)^{2}(x) d x+\int_{0}^{\tau} \frac{\beta}{2}\left(u_{\epsilon}^{2}(t)+1\right)\left(\int_{0}^{1} z^{2} d x\right) d t
$$

and Gronwall's inequality implies,

$$
\begin{aligned}
\int_{0}^{1} z^{2}\left(x, u_{\epsilon}\right) d x & \leq\left\|z^{0}\right\|_{L^{2}([0,1])}^{2} \exp \frac{\beta}{2}\left(\left\|u_{\epsilon}(t)\right\|_{L^{2}(0, T)}^{2}+T\right) \\
& \leq\left\|z^{0}\right\|_{L^{2}([0,1])}^{2} \exp \left(\frac{\beta}{2} J(0)+\frac{\beta T}{2}\right)
\end{aligned}
$$

which is bounded independently of $M$.

And by the same method, one obtain

$$
\begin{aligned}
\int_{0}^{1} p^{2}\left(x, u_{\epsilon}\right) d x & \leq\|p(T)\|_{L^{2}([0,1])}^{2} \exp \frac{\beta}{2}\left(\left\|u_{\epsilon}(t)\right\|_{L^{2}(0, T)}^{2}+T\right) \\
& \leq\|p(T)\|_{L^{2}([0,1])}^{2} \exp \left(\frac{\beta}{2} J(0)+\frac{\beta T}{2}\right)
\end{aligned}
$$

which is also bounded independently of $M$.

From (39) and for $\omega \subset] 0,1[$, we obtain

$$
\begin{aligned}
u_{\epsilon}(t) & \leq\left|u_{\epsilon}(t)\right| \\
& \leq \frac{2 \beta}{\epsilon}\left(\int_{0}^{1} z^{2} d x\right)^{1 / 2}\left(\int_{0}^{1} p^{2} d x\right)^{1 / 2} \\
& \leq M_{1}
\end{aligned}
$$

where $M_{1}$ is independent of $M$.

For $M>M_{1}$, (49) becomes $u_{\epsilon}(t)=\frac{2 \beta}{\epsilon}\left\langle\chi_{\omega} \bar{z}(x, t) ; \chi_{\omega} p(t)\right\rangle_{L^{2}(\omega)}$. 
Corollary 2.10. An optimal control $u_{\epsilon}$, the corresponding state $\bar{z}$, and the adjoint state $p$ are necessarily linked by the following relations:

$$
\begin{cases}u_{\epsilon}(t)=\frac{2 \beta}{\epsilon}\left\langle\chi_{\omega} \bar{z}(x, t) ; \chi_{\omega} p(t)\right\rangle_{L^{2}(\omega)} & \\ \frac{\partial \bar{z}}{\partial t}=\lambda \frac{\partial^{2} \bar{z}}{\partial x^{2}}-u(t) \beta \bar{z} & ] 0,1[\times] 0, T[ \\ -\frac{\partial p}{\partial t}=\lambda \frac{\partial^{2} p}{\partial x^{2}}-u(t) \beta p & ] 0,1[\times] 0, T[ \\ \bar{z}(x, 0)=z^{0}(x) & ] 0,1[ \\ p(x, T)=\left(\bar{z}(T)-\chi_{\omega}^{*} z^{d}\right) & ] 0,1[ \\ \bar{z}(0, t)=\bar{z}(1, t)=0 & ] 0, T[ \\ p_{n}(0, t)=p_{n}(1, t)=0 & ] 0, T[\end{cases}
$$

The computation of an optimal control, solution of the problem (24), can be realized by the following formula

$$
\begin{cases}u_{n+1}(t) & =-2 \beta n\left\langle\chi_{\omega} z_{n}(x, t) ; \chi_{\omega} p_{n}(t)\right\rangle_{L^{2}(\omega)} \\ u_{1} & =0\end{cases}
$$

where $z_{n}$ is the solution of (48) associated with $u_{n}$, and $p_{n}$ is the solution of the adjoint equation

$$
\begin{cases}-\frac{\partial p_{n}}{\partial t}=\lambda \frac{\partial^{2} p_{n}}{\partial x^{2}}-u_{n}(t) \beta p_{n} & ] 0,1[\times] 0, T[ \\ p_{n}(T)=\left(\bar{z}_{n}(T)-\chi_{\omega}^{*} z^{d}\right) & ] 0,1[ \\ p_{n}(0, t)=p_{n}(1, t)=0 & ] 0, T[\end{cases}
$$

which allows to consider the following algorithm

Step 1 : Initialize system data.

$$
\| \begin{aligned}
& z^{0}, u_{0}=0, \text { and desired state } z^{d} . \\
& \text { Define threshold accuracy } \varepsilon, \text { subregion } \omega \text { and time } T .
\end{aligned}
$$

Algorithm 2.11. Step 2 : Until $\left\|u_{n+1}-u_{n}\right\| \leq \varepsilon$ repeat

$$
\begin{aligned}
& \text { Solve the equation (48), which gives } z_{n}(t) \text {. } \\
& \text { Solve the equation (56), which gives } P_{n}(t) \text {. } \\
& \text { Compute } u_{n+1} \text { by the formula (55). }
\end{aligned}
$$

Step 3: The control $u_{n}$ steers the system (48) to the desired state $z^{d}$ at time T.

\subsection{Simulations}

Consider the system (48), with $\lambda=\beta=0.01$, and the problem (25). We take $z^{0}(x)=2 x\left(1-x^{3}\right)$, and the desired state $z^{d}(x)=0.94 x(x-1)(x+1)$.

Applying the previous algorithm for different subregions, we obtain:

Case of $\omega=] 0.25,0.55[$, and $T=1$,Figure 7 shows how the reached state (solid line) is very close to the desired one (dotted line) on $\omega$. The desired state is obtained with error $\mathcal{E}=\left\|\bar{z}(T)-z^{d}\right\|_{L^{2}(\omega)}^{2}=3.09 \times 10^{-4}$ and cost $\left\|u_{4}\right\|^{2}=1.53 \times 10^{-5}$.

Case of $\omega=] 0,0.2[$, and $T=2$,Figure 9 shows how the reached state (solid line) is very close to the desired one (dotted line) on $\omega$. The desired state is obtained with error $\mathcal{E}=\left\|\bar{z}(T)-z^{d}\right\|_{L^{2}(\omega)}^{2}=6.01 \times 10^{-4}$, and cost $\left\|u_{4}\right\|^{2}=4.1 \times 10^{-6}$. 


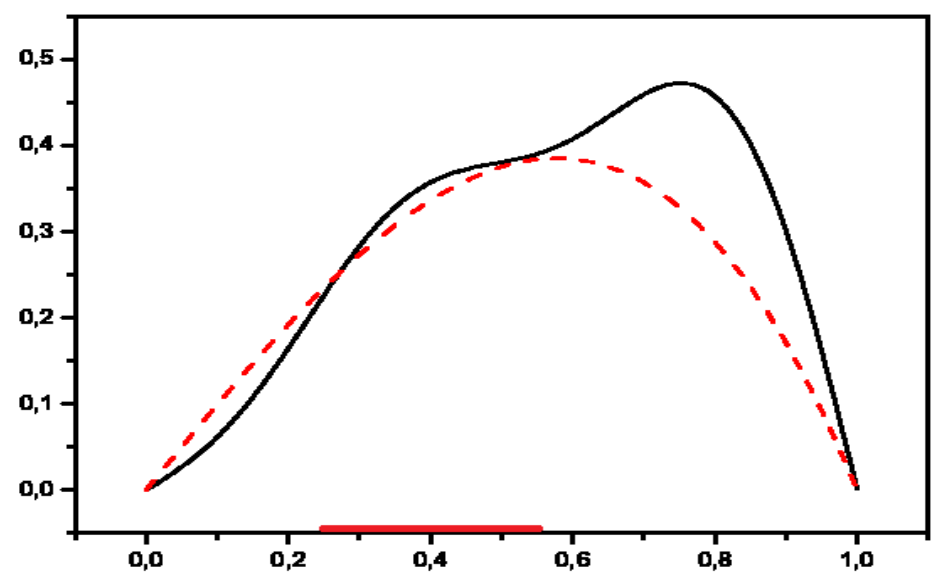

Figure 7. Desired (...) and final state $(-)$ on $\omega$.

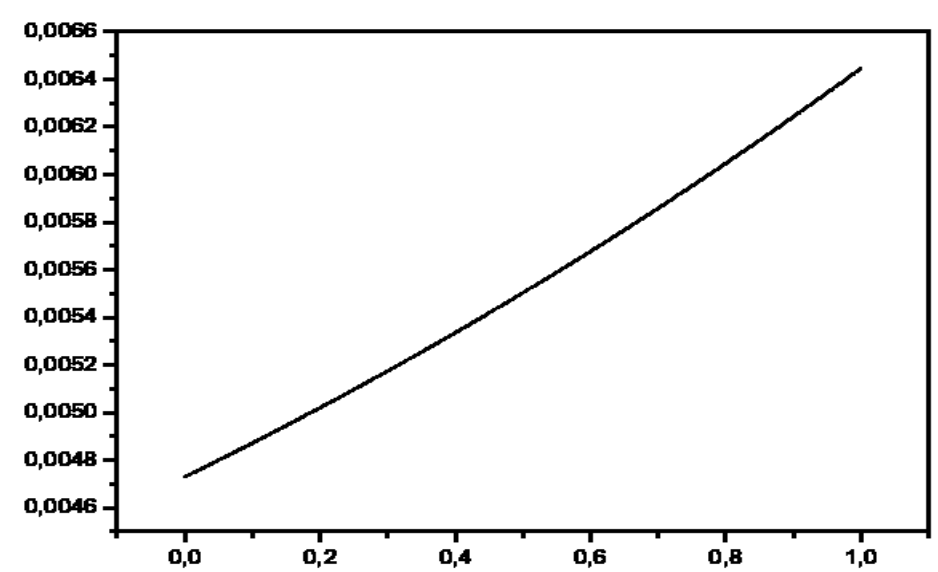

Figure 8. Evolution of the control function $u_{4}^{*}$ on $[0,1]$.

Case of $\omega=\Omega=] 0,1[$, and $T=2$, Figure 11 shows how the reached state (solid line) is very close to the desired one (dotted line) on $\Omega$. The desired state is obtained with error $\mathcal{E}=\left\|\bar{z}(T)-z^{d}\right\|_{L^{2}(\omega)}^{2}=1.8 \times 10^{-2}$, and cost $\left\|u_{4}\right\|^{2}=1.47 \times 10^{-4}$. 


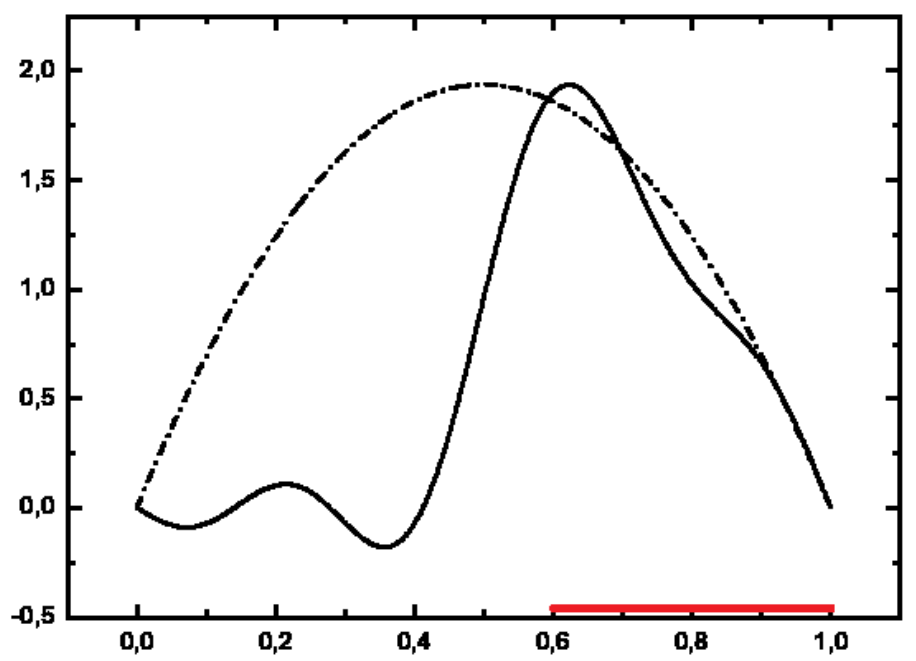

Figure 9. Desired (...) and final state $(-)$ on $\omega$.

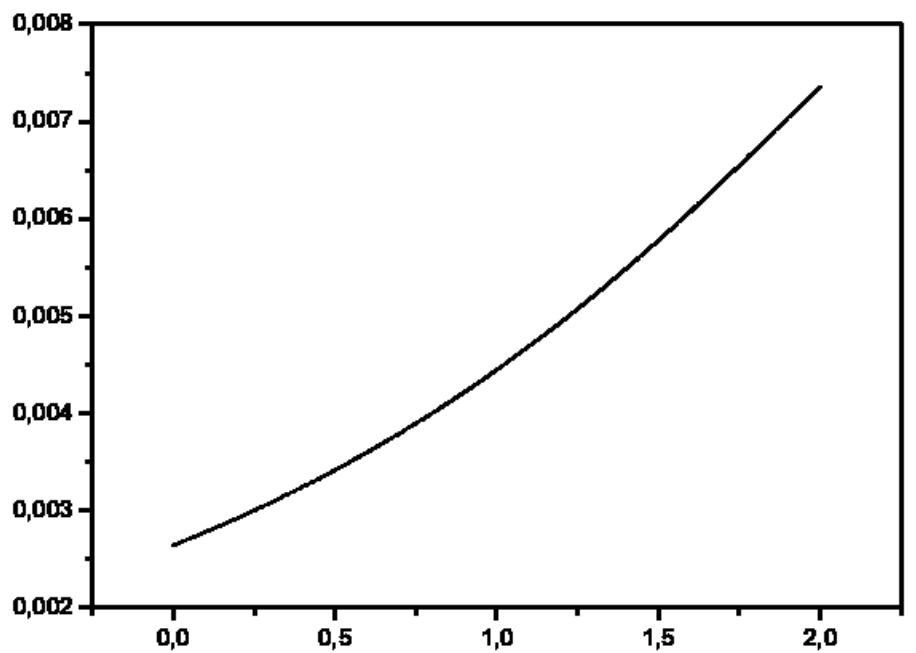

FiguRE 10. Evolution of the control function $u_{4}^{*}$ on $[0,2]$.

\subsection{Conclusion}

The problem of regional minimum energy for distributed bilinear parabolic systems is considered. The solution of this problem is obtained as limit of solutions of regional quadratic control problems. The two cases of bounded and unbounded controls were discussed. Moreover, we developed a numerical approach that led to implicit formula for optimal control. The obtained results are successfully tested through numerical simulations. For future research, several questions are still open. This is the cases of characterization of controllable subregions, the link between the cost of optimal regional controllability and the area of the subregion. The extensions of the above results to bilinear hyperbolic systems are under consideration. 


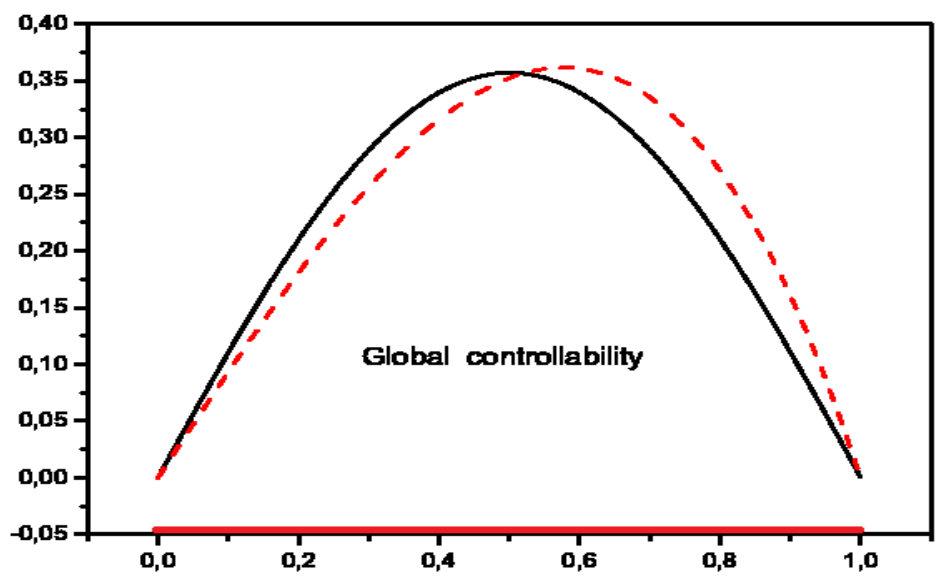

Figure 11. Desired (...) and final state $(-)$ on $\Omega$.

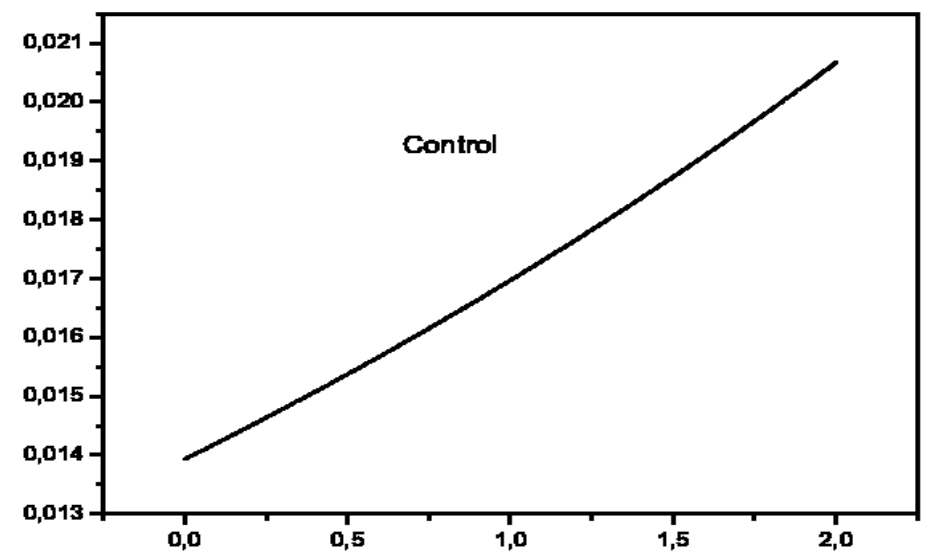

Figure 12. Evolution of the control function $u_{4}^{*}$ on $[0,2]$.

\section{REFERENCES}

[1] J. L. Lions.

Contrôle optimal des systèmes gouvernés par des équations aux dérivées partielles (1966), Dunod . Paris.

[2] J. L. Lions and E. Magenes.

Problèmes aux limites non homogènes et applications. Dunod, 1968, Vol. 1 et 2.

[3] J. Ball, J. E. Marsden and M. Slemrod.

Controllability for Distributed Bilinear systems. SIAM J. on control and opt., 1982, 40, 4, pp. 575-597.

[4] H. Brezis.

'Analyse fonctionnelle: théorie et application' (Masson, 1983).

[5] A. Pazy.

Semigroups of linear operators and applications to partial differential equations. Springer-Verlag, 1983, New-York.

[6] N. El Alami.

Algorithmes pour la commande optimale avec critère quadratique des systèmes bilinéaires. Lecture Notes in Control and Information Sciences, 1988, 111, pp. 432-444.

[7] A. El Jai, A.J. Pritchard, M. C. Simon and E. Zerrik.

Regional controllability of distributed systems, International Journal of Control, 1995, 62, 6, pp. 1351-1365. 
[8] R.F. Curtain and H. Zwart.

An introduction to infinite dimensional linear systems theory/ Texts in applied mathematics (1995), Springer Verlag 21.

[9] T. Kato.

Perturbation theory for linear operators. Springer Verlag, 1995, Berlin Heidelberg.

[10] S. Lenhart.

Optimal control of a convective-diffusive fluid problem. Math. Models Method Appl. Sci., 1995, 5, 2, pp. 225-237.

[11] M. E. Bradeley and S. Lenhart.

Bilinear optimal control of a Kirchhoff plate to a desired profile', Journal of Optimal control applications \& methods, 1997, 18, 3, pp. 217-226.

[12] M. E. Bradeley, S. Lenhart and J. Yong.

Bilinear Optimal control of the Velocity term in a Kirchhoff plate equation. Journal of Mathematical Analysis and Applications, 1999, 238, pp. 451-467.

[13] S. Lenhart and M. Liang. Bilinear optimal control for a wave equation with viscous damping, Houston J. Math., 2000, 26, pp. 575-595.

[14] M. E. Bradeley and S. Lenhart.

Bilinear spatial control of the velocity term in a Kirchhoff plate equation, Electronic Journal of Differental Equations, 2001, 27, pp. 1-15.

[15] A.Y. Khapalov.

On bilinear controllability of the parabolic equation with the reaction-diffusion term satisfying Newton's Law, the special issue of the J; Comput. Appl. Math;, dedicated to the memory of J.-L. Lions, 2002, 21, pp. 1-23.

[16] A.Y. Khapalov.

Global non-negative controllability of the semilinear parabolic equation governed by bilinear control, ESAIM: COCV, 2002, 7 , pp. 269-283.

[17] A. Addou and A. Benbrik.

'Existence and uniqueness of optimal control for a distributed-parameter bilinear system', Journal of dynamical and control systems, 2002, 8, (2), pp. 141-152.

[18] E. Zerrik, M. Ouzahra and K. Ztot.

Regional stabilization for infinite bilinear systems, IEE Cont. Theory. Appl., 2004, 151(1), 109-116.

[19] H. R. Joshi.

Optimal control of the convective velocity coefficient in a parabolic problem, Nonlinear Analysis, 2005, 63, pp. 1383-1390

[20] K. Ito and K. Kunisch.

Optimal bilinear control of an abstract Schrodinger equation. SIAM J. Control Optim. 2007, 46(1) : (electronic), pp 274-287

[21] E. Zerrik and A. Kamal.

Output controllability for semi linear distributed parabolic system. Journal of Dynamical and Control system, 2007, vol. 13, no. 2,: 289-306.

[22] E. Zerrik, R. Larhrissi and H. Bourray.

An output controllability problem for semi linear distributed hyperbolic system.

Journal of Applied Mathematics and Computer Science, 2007, vol. 17, no. 4, pp. 437-448.

[23] E. Zerrik and M. Ould Sidi.

Regional controllability of linear and semi linear hyperbolic systems.Int. Journal of Math. Analysis, 2010, 4,44, pp. $2167-2198$.

[24] E. Zerrik and M. Ould Sidi.

'An output controllability of bilinear distributed system', International Review of Automatic Control', 2010, 3, 5, pp. 466-473.

[25] H. Brezis.

Functional Analysis, Sobolev Spaces and Partial Differential Equations (2010). Springer .

[26] E. Zerrik and M. Ould Sidi.

Regional Controllability for Infinite Dimensional Distributed Bilinear Systems. Int. Journal of control, 2011, 84, 12, pp. 21082116.

[27] K. Ztot, E. Zerrik and H. Bourray.

Regional control problem for distributed bilinear systems. Int. J. Appl. Math. Comput. Sci., 2011, 21, 3, pp. 499-508. 